\title{
The Dynamical State of The Serpens South Filamentary Infrared Dark Cloud
}

\author{
Tomohiro Tanaka ${ }^{1}$, Fumitaka Nakamura ${ }^{2,3}$, Yuya Awazu ${ }^{1}$, Yoshito Shimajiri ${ }^{3}$, Koji Sugitani ${ }^{4}$, \\ Toshikazu Onishi ${ }^{1}$, Ryohei Kawabe ${ }^{2,3,5}$, Hiroshige Yoshida ${ }^{6}$, Aya E. Higuchi ${ }^{5}$
}

\begin{abstract}
We present the results of $\mathrm{N}_{2} \mathrm{H}^{+}(J=1-0)$ observations toward Serpens South, the nearest cluster-forming, infrared dark cloud. The physical quantities are derived by fitting the hyperfine structure of $\mathrm{N}_{2} \mathrm{H}^{+}$. The Herschel and 1.1-mm continuum maps show that a pc-scale filament fragments into three clumps with radii of $0.1-0.2 \mathrm{pc}$ and masses of $40-230 M_{\odot}$. We find that the clumps contain smaller-scale $(\sim 0.04$ pc) structures, i.e., dense cores. We identify 70 cores by applying CLUMPFIND to the $\mathrm{N}_{2} \mathrm{H}^{+}$data cube. In the central cluster-forming clump, the excitation temperature and line-width tend to be large, presumably due to protostellar outflow feedback and stellar radiation. However, for all the clumps, the virial ratios are evaluated to be $0.1-0.3$, indicating that the internal motions play only a minor role in the clump support. The clumps exhibit no free-fall, but low-velocity infall, and thus the clumps should be supported by additional forces. The most promising force is the globallyordered magnetic field observed toward this region. We propose that the Serpens South filament was close to magnetically-critical and ambipolar diffusion triggered the cluster formation. We find that the northern clump, which shows no active star formation, has a mass and radius comparable to the central cluster-forming clump, and therefore, it is a likely candidate of a pre-protocluster clump. The initial condition for cluster formation is likely to be a magnetically-supported clump of cold, quiescent gas. This appears to contradict the accretion-driven turbulence scenario, for which the turbulence in the clumps is maintained by the accretion flow.
\end{abstract}

Subject headings: ISM:structure — ISM: clouds — ISM: kinematics and dynamics — stars: formation

\footnotetext{
${ }^{1}$ Department of Physical Science, Osaka Prefecture University, Gakuen f1-1, Sakai, Osaka 599-8531, Japan

${ }^{2}$ National Astronomical Observatory, Mitaka, Tokyo 181-8588, Japan; fumitaka.nakamura@nao.ac.jp

${ }^{3}$ Nobeyama Radio Observatory, Minamimaki, Minamisaku, Nagano 384-1805, Japan

${ }^{4}$ Graduate School of Natural Sciences, Nagoya City University, Mizuho-ku, Nagoya 467-8501, Japan

${ }^{5}$ Joint ALMA Observatory, Alonso de Cordova 3107 OFC 129, Vitacura, Chile

${ }^{6}$ Caltech Submillimeter Observatory, 111 Nowelo St. Hilo HI 96720 U.S.A.
} 


\section{Introduction}

Infrared Dark Clouds (IRDCs) are cold, dense regions of molecular clouds seen as extinction features against bright mid-infrared galactic backgound (e.g., Rathborne et al. 2006; Peretto \& Fuller 2009; Pillai et al. 2006). They were first identified on the basis of observations of ISO and MSX satellites (Perault et al. 1996; Egan et al. 1998). Since the abundant discovery by the Spitzer Space telescope, IRDCs have been paid attention as promising sites to study the earliest phases of star cluster formation because their volume and surface densities resemble those of the nearby active cluster-forming clumps like NGC 1333 and $\rho$ Oph (Kauffmann \& Pillai 2010; Hernandez \& Tan 2011). Recent observations have revealed that the IRDCs often show elongated or filamentary shapes and appear to fragment into dense clumps (e.g., Miettinen 2012). The observations also suggest that the self-gravity is likely to play an important role in the dynamics of the clumps in IRDCs (e.g., Pillai et al. 2006, 2011; Rygl et al. 2010; Miettinen 2012; Busquet et al. 2013). Global inflow motions toward the IRDC filaments have also been reported (Schneider et al. 2010). These observations indicate that kinematics and dynamics of IRDCs and their substructures can be well characterized by the combination between the dust continuum and molecular line observations. Although extensive observations towards IRDCs have just started recently, our current knowledge on the physical properties of IRDCs remain very limitted.

In order to further characterize the physical properties of IRDCs and to constrain how clusters form in IRDCs, we carried out mapping observations toward a nearby filamentary IRDC, Serpens South, which was discovered by the Spitzer observations (Gutermuth et al. 2008). The IRDC associated with the Serpens South cluster is the nearest IRDC that shows a sign of active cluster formation. An interesting characteristic of the cluster is its extremely-high fraction of protostars. In the central region, the number fraction of protostars (Class I) relative to the Young Stellar Objects (YSOs) detected by the Spitzer telescope (Class I+Class II) reaches about $80 \%$ (Gutermuth et al. 2008). This fraction is largest among the cluster-forming regions known within the nearest $400 \mathrm{pc}$ (see Evans et al. 2009). For example, the Serpens Main Cloud, a nearby embedded cluster having the size comparable to Serpens South, have the protostar fraction of about $60 \%$, about two thirds of Serpens South. Another nearby embedded cluster, NGC 1333, has the protostar fraction of about $30 \%$, less than a half of Serpens South. Very recently, Bontemps et al. (2010) and Könyves et al. (2010) discovered 7 Class 0 protostars in the Serpens South IRDC on the basis of the Herschel observations, all of which haven't been identified by the Spitzer observations. These observations strongly suggest that Serpens South is in the very early phase of star cluster formation. We note that the distance to Serpens South is somewhat uncertain in the range from 260 and 700 pc (see the discussion of Bontemps et al. 2010). In this paper, we adopt the distance of 415 pc (Dzib et al. 2010) because our preliminary analysis on the basis of the near-infrared observations implies a relatively larger distance of $\gtrsim 400 \mathrm{pc}$.

Recently, Nakamura et al. (2011) performed ${ }^{12} \mathrm{CO}(J=3-2)$ mapping observations toward the Serpens South IRDC using the ASTE 10-m telescope and discovered that a number of powerfull outflows are blowing out of the central dense clump that is located near the southern edge of a 
long filamentary cloud (André et al. 2010; Bontemps et al. 2010; Arzoumanian et al. 2013). The CO $(J=3-2)$ images have revealed that several collimated outflow lobes are overlapping and interacting with one another, indicating that the very active star formation is ongoing. From the

$\mathrm{H}_{2} v=1-0 \mathrm{~S}(1) 2.122 \mu \mathrm{m}$ image, Teixeira et al. (2012) also identified 10 outflow knots in Serpens South. The main filament appears to be penetrated by a globally-ordered magnetic field, implying that the magnetic field plays a role in the filament formation (Sugitani et al. 2011). Several lessdense filamentary structures also appear to converge toward the main filament and the dense clump. These less-dense filaments may give us clues to uncover the formation process of the cluster (Myers 2009). Recently, Kirk et al. (2013) performed a Mopra observation toward Serpens South using several dense gas tracers such as $\mathrm{N}_{2} \mathrm{H}^{+}(J=1-0), \mathrm{HCO}^{+}(J=1-0)$, and $\mathrm{H}^{13} \mathrm{CO}^{+}(J=1-0)$. The optically-thick lines like $\mathrm{HCO}^{+}(J=1-0)$ showed blue-skewed profiles, indicative of the infall motions along the line-of-sight. They also found a significant velocity gradient in the $\mathrm{N}_{2} \mathrm{H}^{+}$ emission along the southern part of the main filament. They interpreted that the velocity gradient is caused by a mass infall along the filament toward the central cluster.

In this paper, we present the results of the $\mathrm{N}_{2} \mathrm{H}^{+}(J=1-0)$ observations toward the filamentary IRDC, Serpens South, using the Nobeyama 45-m radio telescope, and study the dynamical state of the dense clumps in Serpens South. The details of the observations are described in Section 2. The results of the observations are presented in Section 3. Our $\mathrm{N}_{2} \mathrm{H}^{+}(J=1-0)$ data have a finer angular resolution and higher sensitivity than those of Kirk et al. (2013), and therefore allow us to estimate the quantities by fitting the hyperfine structure of $\mathrm{N}_{2} \mathrm{H}^{+}$. Applying the hyperfine fitting to the $\mathrm{N}_{2} \mathrm{H}^{+}$data cube, we estimate the physical quantities of the dense gas in this region in Section 4, and assess in Section 5 the dynamical state of the main filament that appears to fragment into a few dense clumps. Then, we apply in Section 6 the virial analysis to the dense clumps, and clarify the clump dynamical state. Then, in Section 7, we identify the dense cores using the clumpfind method and derive the physical quantities of the cores. Finally, we summarize our main conclusion in Section 8 ,

\section{Observations and Data}

\subsection{Nobeyama 45-m Radio Telescope}

In January and March 2012, we carried out $\mathrm{N}_{2} \mathrm{H}^{+}[J=1-0$; the rest frequency of the main component $\left.\left(F_{1} F \rightarrow F_{1}^{\prime} F^{\prime}=23 \rightarrow 12\right)=93.173777 \mathrm{GHz}\right]$ observations toward the Serpens South cluster with the 25-element focal plane receiver BEARS equipped in the 45-m telescope at NRO. In Figure 1, the observed area is indicated by a dashed box on the three-color Herschel image. The observation box size is $9 . ' 5 \times 11^{\prime}$ with the map center of (R.A. [J2000], Decl. [J2000]) $=(18: 30: 1.85$, -02:02:28.6). At $93 \mathrm{GHz}$ band, the telescope has a Full-Width-at-Half-Maximum (FWHM) beam size of $18^{\prime \prime}$ and a main beam efficiency of $\eta=0.51$. The beam separation of the BEARS is $41.1^{\prime \prime}$ on the plane of the sky (Sunada et al. 2000; Yamaguchi et al. 2000). At the back end, we used 25 
sets of 1024 channel Auto Correlators (ACs), which have a bandwidth of $8 \mathrm{MHz}$ and a frequency resolution of $7.8 \mathrm{kHz}$, corresponding to a velocity resolution of $0.025 \mathrm{~km} \mathrm{~s}^{-1}$ at $93 \mathrm{GHz}$ (Sorai et al. 2000). The final velocity resolution was binned to $0.05 \mathrm{~km} \mathrm{~s}^{-1}$. The $\mathrm{N}_{2} \mathrm{H}^{+}(J=1-0)$ consists of seven hyperfine components over 4.6 MHz. We determined the observed frequency range such that all the 7 hyperfine components are distributed around the central frequency. During the observations, the system noise temperatures were in the range between 300 and $450 \mathrm{~K}$ in a double sideband. The standard chopper wheel method (Kutner \& Ulich 1981) was used to convert the output signal into the antenna temperature $T_{A} *(\mathrm{~K})$, corrected for the atmospheric attenuation.

The telescope pointing was checked every 1 hour by observing a $\mathrm{SiO}$ maser source, IRC+00363, and the typical pointing offset were $1^{\prime \prime} \sim 3^{\prime \prime}$ during the whole observing period. Our mapping observations were made with the OTF mapping technique (Sawada et al. 2008). We obtained an OTF map with xscan and yscan over 27 boxes and combined them into a single map, in order to reduce the scanning effects. We adopted a convolutional scheme with a spheroidal function (Sawada et al. 2008) to calculate the intensity at each grid point of the final data with a spatial grid size of $7.5^{\prime \prime}$, about a half of the beam size. The resultant effective angular resolution was $24^{\prime \prime}$, corresponding to $0.05 \mathrm{pc}$ at a distance of $415 \mathrm{pc}$. The rms noise level of the final maps was 0.2 $\mathrm{K}$ in $T_{A}^{*}(1 \sigma)$ at a velocity resolution of $0.05 \mathrm{~km} \mathrm{~s}^{-1}$. We adopted the rest frequency of the main hyperfine component to make the data cube. To make the channel map and apply the clumpfind, we used the data cube whose rest frequency is set to that of the isolated hyperfine component.

\subsection{CSO 10.4-m Radio Telescope}

We carried out single-point observations toward the Serpens South cluster in $\mathrm{HCO}^{+}(J=3-2)$ and $\mathrm{H}^{13} \mathrm{CO}^{+}(J=3-2)$ on May 15, 2011 and August 27, 2011, using the 10.4-m telescope of the Caltech Submillimeter Observatory (CSO) at Mauna Kea, Hawaii. The observed points have the following coordinates: (R.A. [J2000], Decl. [J2000]) $=(18: 30: 03.80,-02: 03: 03.9)$ and (18:29:57.40, $-01: 58: 45.9$ ). The former (hereafter, CSO-C) and latter (hereafter, CSO-N) are pointing toward the $1.1 \mathrm{~mm}$ dust continuum emission peaks of the central and northern clumps, respectively, as discussed in Section 3. The observed points are indicated by the crosses in Figure 1. At 270 $\mathrm{GHz}$ band, the telescope has a main beam efficiency of $\eta=0.72$ and a FWHM beam size of $30^{\prime \prime}$, corresponding to $0.06 \mathrm{pc}$ at a distance of $415 \mathrm{pc}$. The observations were made with a positionswitch mode, using a $230 \mathrm{GHz}$ receiver. At the back end, we used a FFTS1 spectrometer with 8192 channels that covers the $1 \mathrm{GHz}$ bandwidth. The frequency resolution of $122 \mathrm{kHz}$ corresponds to the velocity resolution of $0.14 \mathrm{~km} \mathrm{~s}^{-1}$ at $270 \mathrm{GHz}$. During the observations, the system noise temperatures were about $500 \mathrm{~K}$ and $200 \mathrm{~K}$ on May 15 and August 27, respectively, in a double sideband. The rms noise levels of the $\operatorname{HCO}^{+}(J=3-2)$ and $\mathrm{H}^{13} \mathrm{CO}^{+}(J=3-2)$ data were $\Delta T_{\mathrm{rms}} \sim 0.05 \mathrm{~K}$ in $T_{A}^{*}(1 \sigma)$ at a velocity resolution of about $0.3 \mathrm{~km} \mathrm{~s}^{-1}$. The two molecular lines were detected towards CSO-C, whereas none of the molecular lines were detected towards CSO-N. For CSO-N, we observed additional two molecular lines, $\mathrm{CS}(J=5-4)$ and $\operatorname{HCN}(J=3-2)$. We 
could not detected these two line emissions with the same rms noise as that of $\mathrm{HCO}^{+}(J=3-2)$ and $\mathrm{H}^{13} \mathrm{CO}^{+}(J=3-2)$.

\section{3. $\quad 1.1 \mathrm{~mm}$ AzTEC/ASTE Data}

The $1.1 \mathrm{~mm}$ continuum data were taken toward a $\sim 20^{\prime} \times 20^{\prime}$ area centered on the Serpens South cluster with the AzTEC camera mounted on the Atacama Submillimeter Telescope Experiment (ASTE) 10-m telescope. The observed area is the area enclosed by solid lines in Figure 1. The rms noise level was about $10 \mathrm{mJy}^{\text {beam }^{-1}}$ and the effective beam size was $40^{\prime \prime}$ after the FRUIT imaging, which is an iterative mapping method to recover the spatially extended component. The FRUIT method is based on an iterative mapping method applied to the BOLOCAM data reduction pipeline (Enoch et al. 2006). The FRUIT data reduction pipeline is written in IDL and developed by the University of Massachusetts. The FRUIT algorithm is described in Liu et al. (2010) and Shimajiri et al. (2011). The detail of the AzTEC data will be presented in Gutermuth et al. (2013, in preparation).

\subsection{Herschel Data}

We used the version 2.5 fits files of the Herschel archival data of the PACS $160 \mu \mathrm{m}$ and SPIRE 250, 350, and $500 \mu \mathrm{m}$ towards Serpens South. The detail of the data will be described in Sugitani et al. (2013, in preparation). In brief, all the data were convolved to the ones with the angular resolution of $36.3^{\prime \prime}$, and then adopting the grey-body approximation, we performed the spectral energy distribution (SED) fitting at each grid point to derive the physical quantities such as the column density and dust temperature.

\section{Results}

\subsection{Spatial Distribution of the $1.1 \mathrm{~mm}$ Continuum Emission}

Previous observations have demonstrated that the optically-thin dust continuum emission at submillimeter wavelengths provides a powerful diagnostic to constrain the density and temperature structure of molecular clouds (e.g., André et al. 2000). Our 1.1mm dust continuum data also indicate that the dust continuum emission traces well several YSO envelopes and the spatial distribution of the dense and/or warm molecular gas in this region.

In Figure 2, we present the $1.1 \mathrm{~mm}$ continuum emission map taken with the AzTEC camera mounted on the ASTE 10-m telescope. This is essentially the same as Figure 3 of Nakamura et al. (2011). For comparison, the positions of the YSOs identified by the Spitzer observations (Gutermuth et al. 2008) and the positions of the YSOs and prestellar cores identified from the MAMBO $1.3 \mathrm{~mm}$ ob- 
servations (Maury et al. 2011) are indicated in Figures $2 \mathrm{a}$ and 2b, respectively. The dust image was obtained by applying the FRUIT method to recover the spatially-extended component. The resultant effective angular resolution is $\sim 40^{\prime \prime}$.

The dust continuum emission has its maximum towards the Serpens South cluster at the position of (R.A. [J2000], Dec. [J2000]) = (18:30:03.81, -02:03:04), which is embedded in a dusty clump (hereafter referred to as the Serpens South clump or central clump). The Serpens South cluster is located near the southern edge of a long filamentary cloud that was revealed by the Herschel observations (André et al. 2010). The Serpens South clump is also located at the intersection between the two filaments (south-east and south-west filaments) seen in the southern part of the image. At the southern part of the main filament (south-east filament), the main filament appears to fragment into an elongated clump (hereafter, southern clump), at the peak of which a Herschel Class 0 object is located. In the northern part of the map, we found a V-shape clump (hereafter, northern or V-shape clump). Several starless cores and Class 0 sources identified by the Herschel observations can be recognized as compact structures even in our FRUIT map. Asides from the main filamentary structure, there are a couple of faint filamentary structures that appear to converge toward the Serpens South clump. These faint filaments can also be recognized in the Spitzer image (see Gutermuth et al. 2008; Nakamura et al. 2011). The detail of the 1.1-mm continuum image will be presented in Gutermuth et al. (2013, in preparation).

\subsection{Spatial Distribution of $\mathbf{N}_{2} \mathbf{H}^{+}(J=1-0)$ Emission}

Figure 3 a shows the $\mathrm{N}_{2} \mathrm{H}^{+}(J=1-0)$ intensity map toward Serpens South, integrated over the seven components of the hyperfine multiplet, taken with the Nobeyama 45-m telescope. Our

$\mathrm{N}_{2} \mathrm{H}^{+}(J=1-0)$ intensity distribution is in good agreement with that obtained by Kirk et al. (2013) and has a finer angular resolution and higher sensitivity. On average, the integrated $\mathrm{N}_{2} \mathrm{H}^{+}$ intensity closely follows the $1.1 \mathrm{~mm}$ continuum emission, suggesting that the $\mathrm{N}_{2} \mathrm{H}^{+}$emisson is an excellent tracer of cold and dense emission, as is known from previous studies (e.g., Walsh et al. 2007). However, there are a couple of exceptions. For example, the V-shape clump identified by the $1.1 \mathrm{~mm}$ continuum emission is not so prominent in the $\mathrm{N}_{2} \mathrm{H}^{+}$intensity map. In addition, the $\mathrm{N}_{2} \mathrm{H}^{+}$ integrated intensity takes its maximum at (R.A. [J2000], Decl. [J2000]) $=(18: 30: 03.7,-02: 03: 10)$, which is slightly offset from the continuum maximum by $\sim 10^{\prime \prime}$, about a half the NRO $45-\mathrm{m}$ beam size. Since the pointing errors of both the $1.1 \mathrm{~mm}$ dust continuum map and $\mathrm{N}_{2} \mathrm{H}^{+}$are better than a few arcseconds, we consider that the offset is real. However, the offset may be due to the different angular resolutions of both maps.

In addition, the positions of the dust continuum sources identified by the Herschel observations (Bontemps et al. 2010) often show deviations from the peaks of the $\mathrm{N}_{2} \mathrm{H}^{+}$emission. The $\mathrm{N}_{2} \mathrm{H}^{+}$ integrated intensity tends to be weaker toward the Class 0 and Class 0/I candidates (the black crosses in Fig. 3a), in particular toward the three sources located in the northern part of the image. The $\mathrm{N}_{2} \mathrm{H}^{+}$integrated intensity is very weak toward the Herschel starless cores (the white crosses 
in Fig. 3a). The starless core located at the southwest edge of the image appears almost devoid of $\mathrm{N}_{2} \mathrm{H}^{+}$emission. There are two possibilities to explain these descrepancies. One possibility is that these dust cores are chemically young and that the abundance of a late-stage molecule, $\mathrm{N}_{2} \mathrm{H}^{+}$, is still relatively low. Another possibility is that $\mathrm{N}_{2} \mathrm{H}^{+}$molecules tend to be destroyed by $\mathrm{CO}$ and the $\mathrm{N}_{2} \mathrm{H}^{+}$abundance has become small.

Very recently, we carried out CCS $\left(J_{N}=2_{1}-1_{0}\right), \operatorname{CCS}\left(J_{N}=4_{3}-3_{2}\right)$, and $\operatorname{CCS}\left(J_{N}=7_{7}-6_{5}\right)$ observations toward a $20^{\prime} \times 10^{\prime}$ area including the $\mathrm{N}_{2} \mathrm{H}^{+}$mapping area, and found that the CCS emissions tend to be stronger towards the starless dust cores identified by Herschel. Therefore, we interpret that the weak $\mathrm{N}_{2} \mathrm{H}^{+}$emission towards the starless cores implies that the cores are chemically young. We will present the results of the CCS observations in a forthcoming paper.

In Figure 3b, we compare the $\mathrm{N}_{2} \mathrm{H}^{+}$integrated intensity map with the ${ }^{12} \mathrm{CO}(J=3-2)$ integrated intensity map obtained by Nakamura et al. (2011). The strong CO emission is concentrated in the central clump that has the strongest $\mathrm{N}_{2} \mathrm{H}^{+}$emission. As shown by Nakamura et al. (2011), the $\mathrm{CO}$ emission represents the protostellar outflows blowing out of the central clump. The CO emission is more extended than the dense gas traced by $\mathrm{N}_{2} \mathrm{H}^{+}$, implying that the outflows have broken through the dense clump.

The $\mathrm{N}_{2} \mathrm{H}^{+}$velocity channel maps are presented in Figure 4, which shows that the main filamentary structure is running from south to north. The filament has a large-scale velocity gradient of about $0.5 \mathrm{~km} \mathrm{~s}^{-1} \mathrm{pc}^{-1}$ along its axis. The filament contains several velocity components, implying the existence of the internal structures. Figure 5 illustrates an example of the $\mathrm{N}_{2} \mathrm{H}^{+}$line profile showing two velocity components, where the rest frequency of the strongest hyperfine component is set to the velocity center. The profile presented is averaged in the $30^{\prime \prime} \times 30^{\prime \prime}$ area centered on the position (R.A. [J2000], Decl. [J2000]) $=(18: 30: 04.4,-02: 04: 23.5)$ and the velocity resolution is set to $0.05 \mathrm{~km} \mathrm{~s}^{-1}$. The isolated line at $V_{\mathrm{LSR}} \sim-1 \mathrm{~km} \mathrm{~s}^{-1}$ shows two velocity components with about $1 \mathrm{~km} \mathrm{~s}^{-1}$ separation. Such structure qualitatively resembles those of the B213 filament in Taurus (Hacar et al. 2013). At the velocity of $7.0 \mathrm{~km} \mathrm{~s}^{-1}$, the central clump contains two filamentary structures (shown with the dashed curves). At the velosity of $7.8-7.9 \mathrm{~km} \mathrm{~s}^{-1}$, the arc-like filamentary structure (shown with the dashed curve) can be recognized in the north-east of the central clump.

\section{4. $\mathbf{N}_{2} \mathbf{H}^{+}$Line Analysis}

The $\mathrm{N}_{2} \mathrm{H}^{+}(J=1-0)$ consists of seven hyperfine components over 4.6 MHz. Following Caselli et al. (2002) and Di Francesco et al. (2004), we derive the total line optical depth $\left(\tau_{\text {tot }}\right)$, line width $(\Delta V)$, line-of-sight velocity $\left(V_{\mathrm{LSR}}\right)$, excitation temperature $\left(T_{\mathrm{ex}}\right)$, and column density

$\left(N_{\mathrm{N}_{2} \mathrm{H}^{+}}\right)$by fitting all seven hyperfine components with multiple Gaussian components under the assumption that all the seven hyperfine lines have a single line width and excitation temperature. The rest frequencies and intrinsic relative intensities of the hyperfine components are taken from 
Caselli et al. (1995) and Tiné et al. (2000), respectively. For the hyperfine fitting, we reconstructed the data cube of the intensity with a spatial grid size of $15^{\prime \prime}$ and velocity resolutoin of $0.1 \mathrm{~km} \mathrm{~s}^{-1}$ by applying the convolutional scheme with a spheroidal function, so that the data rms noise level is reduced to $\Delta T_{A}^{*} \sim 76 \mathrm{mK}$ and the hyperfine fitting procedure is more reliable. The effective angular resolution of the data is $37^{\prime \prime}$, comparable to the AzTEC $1.1 \mathrm{~mm}$ and Herschel images. For the following analysis, we use the hyperfine fitting data at all the pixels above the $2 \sigma$ levels for the fitted peak antenna temperature of the $\mathrm{N}_{2} \mathrm{H}^{+}$weakest hyperfine component.

Table 1 lists the mean, rms, maximum, and minimum values of $\Delta V, V_{\mathrm{LSR}}, T_{\mathrm{ex}}$, and $\tau_{\text {tot }}$ obtained from the hyperfine fitting. Here, we compute these values by dividing the observed area into three parts: north (Decl. [J2000] $\geq-02: 02: 02.4)$, center $(-02: 02: 02.4 \geq$ Decl. [J2000] $\geq$ -02:05:32.5), and south (Decl. [J2000] $\leq-02: 05: 32.5$ ), because these three parts appear to be separated in the velocity channel maps presented in Figure 4. The northern, central, and southern parts contain the northern, central, and southern clumps, respectively (see Figure 2b).

In the following, we briefly describe the spatial distributions of these physical quantities obtained by the hyperfine fitting.

\subsection{Column Densities and Abundances}

We estimate the $\mathrm{H}_{2}$ column densities by applying the spectral energy distribution (SED) fitting using the Herschel 160, 250, 350, and $500 \mu \mathrm{m}$ data. The detail of the analysis will be described in Sugitani et al. (2013, in preparation). For the SED fitting, we used the gas-to-dust ratio of 100 and the dust opacity per unit mass of $\kappa_{\nu}=0.005(1.3 \mathrm{~mm} / \lambda)^{\beta} \mathrm{cm}^{2} \mathrm{~g}^{-1}$ with $\beta=2$. These parameters are the same as those adopted by Könyves et al. (2010).

Figure $\left[6\right.$ a shows the $\mathrm{H}_{2}$ column density distribution in Serpens South obtained from the Herschel SED fitting. To compare $\mathrm{N}_{\mathrm{H}_{2}}$ with the $\mathrm{N}_{2} \mathrm{H}^{+}$data, the $\mathrm{H}_{2}$ column density data were remapped into the grid with the same pixels as that of the $\mathrm{N}_{2} \mathrm{H}^{+}$data. The estimated column density is essentially the same as that estimated by André et al. (2010) because the mass per unit length, line mass, is almost equal to their estimate of about $400 \mathrm{M}_{\odot} \mathrm{pc}^{-1}$ at a distance of $415 \mathrm{pc}$. The $\mathrm{N}_{2} \mathrm{H}^{+}$ column densities are calculated by using the formula given in Caselli et al. (2002). We used the optically-thin (thick) formula when the optical depth of the strongest line is smaller (larger) than unity, to take into account the effect of the optical depth. The obtained column density distribution of $\mathrm{N}_{2} \mathrm{H}^{+}$is presented in Figure 6b. Our hyperfine analysis reveals that the V-shape clump which has relatively weak emission in the velocity integrated intensity map has a large column density peak. This is due to the fact that the optical depths of the $\mathrm{N}_{2} \mathrm{H}^{+}$lines presented in Figure 6re are largest toward the V-shape clump. For comparison, we also show the spatial distribution of the excitation temperature $T_{\mathrm{ex}}$ in Figure 6d. The excitation temperature tends to be higher toward the central cluster-forming clump. In the northern clump, the excitation temperature tends to be as low as about $4 \mathrm{~K}$, in spite of the high column density. We note that at the position of the 
dust continuum emission peak (CSO-N), high-transition molecular lines such as CS $(J=5-4)$, $\mathrm{HCO}^{+}(J=3-2), \mathrm{H}^{13} \mathrm{CO}^{+}(J=3-2)$, and $\mathrm{HCN}(J=3-2)$ were not detected with our CSO observations. These lines are not likely to be sufficiently excited due to either low temperatures or low densities, or both.

Figure 6e shows the $\mathrm{N}_{2} \mathrm{H}^{+}$fractional abundance across Serpens South. The mean fractional abundance of this region is estimated to be $X_{\mathrm{N}_{2} \mathrm{H}^{+}} \simeq 2.5 \times 10^{-10}$, which is similar to the values derived for other dark clouds; e.g., $X_{\mathrm{N}_{2} \mathrm{H}^{+}} \approx 10^{-10}$. For example, Di Francesco et al. (2004) and Friesen et al. (2010) estimated the $\mathrm{N}_{2} \mathrm{H}^{+}$fractional abundances of $1.3 \times 10^{-10}$ and $5.6 \times 10^{-10}$ for the Ophiuchus A and B2 regions, respectively. Figure 6r shows that the $\mathrm{N}_{2} \mathrm{H}^{+}$fractional abundance varies by a factor of a few with the lower values of around $1.5 \times 10^{-10}$ at the $1.1 \mathrm{~mm}$ continuum peak, the northern part of the central clump, and the south-west filament, and the higher values of $(4-5) \times 10^{-10}$ at positions in the eastern and southern parts of the central clump and the northern V-shape clump. Table 2 lists the mean, rms, minimum, and maximum of the $\mathrm{N}_{2} \mathrm{H}^{+}$fractional abundances presented in Figure 6.

In Figure 17 the fractional abundances $X_{\mathrm{N}_{2} \mathrm{H}^{+}}$are presented as a function of the $\mathrm{H}_{2}$ column density. Figure 7 indicates that the $\mathrm{N}_{2} \mathrm{H}^{+}$fractional abundance decreases with increasing $\mathrm{H}_{2}$ column density. For comparison, we plot the best-fit power-law function that is given by

$$
\log X_{\mathrm{N}_{2} \mathrm{H}^{+}}=(-9.66 \pm 0.60)+(-0.237 \pm 0.026) \log \left(N_{\mathrm{H}_{2}} / 10^{23} \mathrm{~cm}^{-2}\right),
$$

with the correlation coefficient of $\mathcal{R}=0.49$. Although the correlation is weak, the dependence of $X_{\mathrm{N}_{2} \mathrm{H}^{+}}$on $N_{\mathrm{H}_{2}}$ is consistent with a theoretical consideration of the ionization fraction in molecular clouds, as also pointed out by Maruta et al. (2010). In molecular clouds, the ionization fraction is determined roughly by the balance between the ionization rate by cosmic rays and the recombination rate of ion and electron (Elmegreen 1979). Here, the ionization and recombination rates are proportional to $n_{n}$ and $n_{i} n_{e}$, respectively, where $n_{n}, n_{i}$, and $n_{e}\left(\approx n_{i}\right)$ are the densities of the neutral gas, positive ion, and electron, respectively. As a result, the ionization fraction decreases with increasing neutral density $n_{n}$, as $n_{i} / n_{n} \propto n_{n}^{-1 / 2}$. The ionization fraction is expected to be proportional to $N_{\mathrm{H}_{2}}^{-1 / 4}$ because $N_{\mathrm{H}_{2}} \propto n_{n}^{1 / 2}$ in a self-gravitating cloud.

\subsection{Centroid Velocities and Line Widths}

Figure 8 a shows a map of the centroid velocity (the first moment map), measured by the isolated hyperfine component $\left(F_{1} F \rightarrow F_{1}^{\prime} F^{\prime}=01 \rightarrow 12\right)$, the second weakest hyperfine component, where we calculated the centroid velocity by integrating the antenna temperatures of the isolated hyperfine component for all the pixels above the $4 \sigma$ noise level. A prominent characteristic of the centroid velocity distribution is a steep velocity change of $0.2-0.3 \mathrm{~km} \mathrm{~s}^{-1}$ near the southern part of the central clump at (R.A. [J2000], Decl.[J2000]) (18:30:05, -02:05:00). There is also a velocity jump of about $0.5 \mathrm{~km} \mathrm{~s}^{-1}$ at the intersection between the main filament and the southwest filament of (R.A. [J2000], Decl. [J2000]) (18:30:00, -02:05:00). In the north-west part of 
the central clump, the centroid velocity takes its maximum at $7.8 \mathrm{~km} \mathrm{~s}^{-1}$, which is shifted about $0.5 \mathrm{~km} \mathrm{~s}^{-1}$ from that of the central clump (about $7.2 \mathrm{~km} \mathrm{~s}^{-1}$ ). The area with this large centroid velocity in the central clump is in good agreement with the position of the prominent redshifted outflow lobe discovered by Nakamura et al. (2011), labeled with "R3", implying that the dense gas is interacting with the redshifted outflow lobe R3 (see Figure 8b).

Figure $8 \mathrm{c}$ shows a map of the one-dimensional (1D) velocity dispersion, $\sigma_{\mathrm{v}, 1 \mathrm{D}}(=\Delta V / \sqrt{8 \ln 2})$, (the second moment map) measured by the isolated hyperfine component. The $1 \mathrm{D}$ velocity dispersion tends to be larger at the central clump where active cluster formation is ongoing. In the central clump the velocity dispersion takes its maximum of about $0.5 \mathrm{~km} \mathrm{~s}^{-1}$. The area having a large velocity dispersion appears to overlap well with the blueshifted and redshifted outflow components blowing out of the central clump (see Figure 8d). In the other areas the velocity dispersion tends to be narrow with a mean of about $0.2-0.3 \mathrm{~km} \mathrm{~s}^{-1}$, which indicates that the internal motions are transonic. In particular, the northern and southern clumps have the narrow velocity dispersions of $0.25 \mathrm{~km} \mathrm{~s}^{-1}$ and $0.2 \mathrm{~km} \mathrm{~s}^{-1}$, respectively. The spatial distribution of the velocity dispersion suggests that the star formation activity has increased the internal turbulent motions in this region.

\section{Dynamical State of Main Filament}

Here, we discuss the dynamical stability of the main filament. An isothermal filament is in dynamical equilibrium when the line mass is equal to the critical value of

$$
l_{\mathrm{cr}}=2 \sigma_{v}^{2} / G,
$$

where $\sigma_{v}$ denotes the three-dimensional velocity dispersion including the thermal contribution (Stodółkiewicz 1963; Ostriker 1964). As mentioned in the previous section, the main filament has a line mass of about $400 M_{\odot} \mathrm{pc}^{-1}$ (see also André et al. 2010). From the $\mathrm{N}_{2} \mathrm{H}^{+}$hyperfine fitting, the mean line width is evaluated to be $0.81 \mathrm{~km} \mathrm{~s}^{-1}$, corresponding to $\sigma_{v} \simeq 0.62 \mathrm{~km} \mathrm{~s}^{-1}$. Thus, the critical line mass of the Serpens South main filament is roughly estimated to be $l_{\text {cr }} \sim$ $180 M_{\odot} \mathrm{pc}^{-1}$, under the assumption that the internal turbulence provides the isotropic pressure to support the filament against the gravity. Therefore, the line mass of the main filament is more than twice the critical value of $180 M_{\odot} \mathrm{pc}^{-1}$, suggesting that the filament is very unstable to radial collapse in the absence of additional support. However, as mentioned in the next Section, rapid global collapse has not been detected in this region. Thus, the filament is likely to be supported by additional forces.

A promissing support mechanism is the magnetic field. Nakamura et al. (1993) discussed that in the persence of longitudinal or helical magnetic field, a filamentary cloud can reach its dynamical equilibrium state even if its line mass exceeds $l_{\text {cr }}$ (see also Fiege \& Pudritz 2000). A rotation around the filament axis also provides the radial suppport (Matsumoto et al. 1994). However, there is no evidence of the rotation for the Serpens South filament. According to Nakamura et al. (1993), a 
longitudinal magnetic field can enhance the gravitational fragmentation with help of the magnetic buoyancy instability, i.e., the Parker instability, and the most unstable wavelength relative to the cloud diameter becomes shorter than that with no magnetic field. The longitudinal component, if exists, should have its strength of

$$
B_{\|} \simeq \sqrt{8 \pi \sigma_{v}^{2} \rho} \sim 200\left(\frac{\sigma_{v}}{0.62 \mathrm{~km} \mathrm{~s}^{-1}}\right)\left(\frac{\rho}{10^{5} \mathrm{~cm}^{-3}}\right)^{1 / 2} \mu \mathrm{G}
$$

to support the filament against the radial collapse (Nakamura et al. 1993). However, there is no clear evidence of the presence of the longitudinal magnetic field in the dense regions.

In contrast, recent near-infrared polarimetric observations have revealed that the main filament is penetrated by the globally-ordered magnetic field that can provide the additional support against the gravity (Sugitani et al. 2011). Therefore, the magnetic field may be roughly perpendicular to the filament. A magnetic field perpendicular to the filament axis can also provide the significant dynamical support against gravity if the field strength is close to the critical value for magnetic support. If the Serpens South filament was penetrated by the nearly critical magnetic field, the fragmentation is likely to have been triggered by the ambipolar diffusion since the filament appears to have already fragmented into several dense clumps. However, since the near-infrared observations tend to trace the intermediate-density envelopes $\left(\sim 10^{4} \mathrm{~cm}^{-3}\right)$ of the clouds, further polarimetric observations such as submillimeter continuum polarimetry are needed to uncover the magnetic field structures in the densest part. We will discuss the magnetic support in more detail in Section 6.3.

\section{Dynamical State of Dense Clump}

\subsection{Clump Virial Analysis}

From the dust continuum map, we can recognize several local peaks along the main filament, as already shown in Section 3.1. In other words, the filament apparently fragments into several pieces. Here, we define the subparsec-size substructures having the local peaks as the clumps. We identified three dense clumps along the main filament: northern (V-shape), central (clusterforming), and southern clumps. The clumps are defined as the regions enclosed by a contour of $5 \times 10^{22} \mathrm{~cm}^{-2}$ on the $\mathrm{H}_{2}$ column density map in each (northern, central, or southern) area presented in Fig. 6. We interpret these clumps as sub-units of star or cluster formation in this region. The threshold column density is somewhat arbitrary. However, our main conclusion given in the present paper are not influenced by the actual value of the threshold column density in the range between $\sim 5 \times 10^{22} \mathrm{~cm}^{-2}$ and $\sim 1 \times 10^{23} \mathrm{~cm}^{-2}$. The clumps also contain substructures that are recognized in the velocity-channel maps of $\mathrm{N}_{2} \mathrm{H}^{+}$presented in Figure 4. We identify the internal structures of the clumps as the cores in the next section. Here, we perform the virial analysis using the Herschel and $\mathrm{N}_{2} \mathrm{H}^{+}$data to investigate the dynamical states of the clumps.

Following Nakamura et al. (2011), we adopt the virial equation of a spherical gas cloud that is 
given by

$$
\frac{1}{2} \frac{\partial^{2} I}{\partial t^{2}}=2 U+W
$$

where $I$ is the moment of inertia, $U$ is the internal kinetic energy, and $W$ is the gravitational energy. In the above equation, the surface pressure term is omitted. A clump is in virial equilibrium when $2 U+W=0$. The internal kinetic energy term $U$ and the gravitational energy term $W$ are expressed as follows:

$$
U=\frac{3 M \Delta V^{2}}{16 \ln 2}
$$

and

$$
W=-a \frac{G M^{2}}{R}\left[1-\left(\frac{\Phi}{\Phi_{\mathrm{cr}}}\right)^{2}\right],
$$

where the values $\Phi$ is the magnetic flux penetrating the clump and $\Phi_{\text {cr }}$ is its critical value above which the clump is fully supported by the the magnetic field against the self-gravity (Nakano 1998). In the discussion of this subsection, we assume $\Phi=0$, which means that the clumps are not supported by the magnetic forces. We will discuss the contribution of the magnetic field to the cloud supoprt in Section 6.3. The value $a$ is a dimensionless parameter of order unity which measures how much the mass distribution deviates from a sphere with the profile of $\rho \propto r^{-2}$ (Bertoldi \& McKee 1992). For example, for a uniform sphere and a centrally-condensed sphere with $\rho \propto r^{-2}$, the dimensionless parameter $a$ is given by $3 / 5$ and 1 , respectively. For the identified clumps, we assume that the effects of the nonspherical mass distribution is negligible. This is a reasonable assumption because the aspect ratios of the clumps identified are not so far from unity and in such cases, the value of $a$ is close to unity (Bertoldi \& McKee 1992). Below, we assume $a$ to be unity because each clump has a more or less centrally-condensed density distribution.

Table 3 summarizes the physical quantities of the three clumps. Here, we estimate the masses and radii from the Herschel column density map. The clump radius is determined by taking the area enclosed by the threshold $\mathrm{H}_{2}$ column density of $5 \times 10^{22} \mathrm{~cm}^{-2}$ and computing the radius of the circle required to reproduce the area. The clump line widths are estimated by taking the intensityweighted average of the one-dimensional velocity dispersion obtained from hyperfine fitting of the $\mathrm{N}_{2} \mathrm{H}^{+}$data. The mass of the northern clump is estimated to be about $200 M_{\odot}$, which is comparable to that of the central cluster-forming clump. In spite of the relatively large mass, only several YSOs are identified in the northern clump. This may imply that the northern clump is likely to be the site of future cluster formation, or star formation may be precluded by some additional clump support. The southern clump has about $40 M_{\odot}$ and is less massive than the other two. The line width of the northern clump is evaluated to be about $0.7 \mathrm{~km} \mathrm{~s}^{-1}$, which is as small as that of the typical molecular cloud core in quiescent regions. The southern clump, with which only a few YSOs appear to be associated, also has a similar line width. On the other hand, the central cluster-forming clump has a larger line width of about $1.3 \mathrm{~km} \mathrm{~s}^{-1}$. This may indicate that the star formation activity enhances the internal motions in the central clump.

For all the clumps, the terms $U$ and $W$ are estimated to be $U \approx 40-110 \mathrm{M}_{\odot} \mathrm{km}^{2} \mathrm{~s}^{-2}$ and 
$W \approx-40 \sim-1000 \mathrm{M}_{\odot} \mathrm{km}^{2} \mathrm{~s}^{-2}$, respectively, which yields the virial ratio, the ratio between $2 U$ and $W$, of $0.1-0.3$. For all the clumps, the virial ratios are estimated to be well below the unity, indicating that the internal turbulent motions play only a minor role in the clump support. This conclusion is inconsistent with the previous estimation by Nakamura et al. (2011), who concluded that the Serpens South clump as a whole is close to quasi-virial equilibrium. The main difference between the two estimations comes from the different line widths. Nakamura et al. (2011) obtained the line width of the central clump using $\mathrm{HCO}^{+}(J=4-3)$. However, this line tends to be optically thick toward the central clump, which may lead to the overestimation of the line width. We believe that the present estimation is more reasonable because $\mathrm{N}_{2} \mathrm{H}^{+}(J=1-0)$ tends to be more optically thin than $\mathrm{HCO}^{+}(J=4-3)$ in the densest part. This cloud as a whole seems to be extremely gravitationally unstable in the absence of the additional support mechanisms such as the magnetic support.

We note that the virial ratios of the clumps are not sensitive to the threshold column density that defines the clump boundary $\left(N_{\text {cr }}\right)$. For example, for the threhold density of $N_{\text {cr }} \lesssim 1 \times 10^{23}$ $\mathrm{cm}^{-2}$, the virial ratios of the northern and central clumps are almost the same as those in Table 3 , For the southern clump whose peak column density is $7 \times 10^{22} \mathrm{~cm}^{-2}$, the virial ratio is almost the same as that in Table 3 as long as $N_{\text {cr }} \lesssim 6 \times 10^{22} \mathrm{~cm}^{-2}$.

The clumps and cores having the small virial ratios have recently been reported by a couple of groups. For example, Li et al. (2012) carried out $\mathrm{NH}_{3}$ observations toward OMC-2 and OMC-3 and found that the clumps in this area have small virial ratios. Pillai et al. (2011) performed the virial analysis of the IRDC clumps and found that the IRDC clumps often have low virial ratios (see also Pillai et al. 2013, in prep.).

\subsection{Global Infall}

As shown above, the cloud as a whole seems not to be supported by the internal turbulent motions, and therefore the global infall motions are expected. In fact, the profile maps of the optically thick lines often show blueshifted part stronger than the redshifted part, i.e., blue-skewed (see Nakamura et al. 2011). Such blue-skewed profiles are probably indicative of global infall motions.

In Figure 9, we present a $\mathrm{HCO}^{+}(J=3-2)$ line profile detected toward CSO-C (in the Serpens South clump), whose coordinate is (R.A. [J2000], Decl, [J2000]) $=(18: 30: 03.80,-02: 03: 03.9)$. At CSO-C, the $\mathrm{HCO}^{+}(J=3-2)$ line is likely to be optically-thick. For comparison, an optically-thin line $\mathrm{H}^{13} \mathrm{CO}^{+}(J=3-2)$ is presented in Figure 9. The $\mathrm{HCO}^{+}(J=3-2)$ line shows a clear blueskewed profile, whereas the $\mathrm{H}^{13} \mathrm{CO}^{+}(J=3-2)$ line shows a single-peak profile. This indicates that the densest part has an infall motion.

Applying a simple model of Myers et al. (1996), we evaluated the infall speed of $0.024 \mathrm{~km} \mathrm{~s}^{-1}$, which is subsonic. If the central clump as a whole is contracting with this subsonic infall speed, the mass infall rate is estiamted to be $\dot{M}=4 \pi R_{\text {clump }}^{2} \rho V_{\text {infall }} \sim 9 \times 10^{-5} M_{\odot} \mathrm{yr}^{-1}$, where $\rho$ is set to 
the mean density in the clump, $\rho \sim 5 \times 10^{-18} \mathrm{~g} \mathrm{~cm}^{-3}$. This implies that the mass of the central clump will be doubled within 2.5 Myr. The estimated mass infall rate is in good agreement with that obtained by Kirk et al. (2013) within a factor of a few.

The estimated infall speed is, however, too slow compared to the free-fall velocity of $V \sim$ $\sqrt{G M / R} \sim 1-2 \mathrm{~km} \mathrm{~s}^{-1}$, corresponding to the FWHM line widths of $2-4 \mathrm{~km} \mathrm{~s}^{-1}$. The clump FWHM line widths of $0.5-1.3 \mathrm{~km} \mathrm{~s}^{-1}$ are smaller than this free-fall velocity. Hence, the additional support mechanism should be needed.

\subsection{Magnetic Support}

As discussed above, the internal turbulent motions play only a minor role in the clump support in this region. However, there is no clear evidence showing the free-fall global infall motions of order of a few $\mathrm{km} \mathrm{s}^{-1}$. The line widths along the line-of-sight are at most $0.5-1 \mathrm{~km} \mathrm{~s}^{-1}$. This implies that the additional support mechanism is needed. The most promising mechanism of the additional support is the magnetic support. Here, we discuss whether the magnetic field plays an important role in the clump support in this region.

Sugitani et al. (2011) carried out the near-infrared (JHKs) polarimetric observations toward Serpens South and found that the Serpens South filament is penetrated perpendicularly to the large-scale ordered magnetic field. The global magnetic field also appears to be curved in the southern part of the main filament. Such morphology is consistent with the idea that the global magnetic field is distorted by gravitational contraction along the main filament toward the central cluster-forming clump. Applying the Chandrasekhar-Fermi method, they estimated the magnetic field strength of about $80 \mu \mathrm{G}$ at the filament envelope with the density of $10^{4} \mathrm{~cm}^{-3}$, where we rescaled the distance to $415 \mathrm{pc}$ and used the smaller line width estimated from our $\mathrm{N}_{2} \mathrm{H}^{+}$data. Assuming an inclination angle of $45^{\circ}$, the magnetic field strength is roughly estimated to be about $120 \mu \mathrm{G}$.

The mean densities of the clumps can be estimated to be about $10^{5} \mathrm{~cm}^{-3}\left[=M_{\mathrm{cl}} /\left(4 \pi R_{\mathrm{cl}}^{3} / 3\right)\right]$. Assuming that the magnetic field strength is proportional to the square root of the density, $B \propto \rho^{1 / 2}$, the strength of the magnetic field associated with the clumps is evaluated to be about $400 \mu \mathrm{G}$. If the clumps have the field strengths of about $400 \mu \mathrm{G}$, the mass-to-magnetic-flux ratios are estimated

to be around unity, close to critical. In other words, the magnetic support is expected to play a crucial role in the clump dynamics and evolution.

According to our estimation, the parent cloud was presumably close to magnetically-critical and either the ambipolar diffusion or mass accretion along the magnetic field lines may have triggerd the slow global infall and eventually the active cluster formation that is observed in the central clump (Nakamura \& Li 2010; Bailey \& Basu 2012). 


\subsection{Clump-Mass-Size Relation}

Recently, Kauffmann et al. (2010) and Kauffmann \& Pillai (2010) analyzed the mass and radius of cloud substructures in several nearby molecular clouds over a wide range of spatial scales $(0.05 \lesssim r /$ pc $\lesssim 3)$ and found that the clumps forming massive stars $\left(M \gtrsim 10 M_{\odot}\right)$ have masses larger than $M \gtrsim 1300 M_{\odot}(r / p c)^{1.33}$, where we rescaled Kauffmann \& Pillai's relation by a factor of 1.5 to take into account the difference in the adopted dust parameters. On the other hand, Krumholz \& McKee (2008) proposed the threshold column density of $1 \mathrm{~g} \mathrm{~cm}^{-2}$ for massive star formation. In Figure 10 we plot the three Serpens South clumps (northern, central, and southern clumps) on the mass-radius diagram with Kauffmann \& Pillai's relation and Krumholz \& McKee's criteria for massive star formation. The northern and central clumps are located above the critical mass-radius relation but below the Krumholz criteria. Thus, we expect that these two clumps are borderline clumps for forming massive stars.

Interestingly, all the Serpens South clumps have column densities comparable to about $10^{23}$

$\mathrm{cm}^{-2}$. Such clumps are the densest ones found among nearby star-forming molecular clouds whose distances are less than about $400 \mathrm{pc}$, except in the Orion molecular cloud, the nearest massive star forming region. In the Orion molecular cloud, a number of clumps have the column densities similar to or larger than the Serpens South clumps (see Figure 1 of Kauffmann et al. 2010). In fact, the clumps containing the most massive core found for small radii in the Perseus and Ophiuchus molecular clouds have the column densities comparable to the Serpens South clumps. Therefore, we expect that the physical conditions of the Serpens South clumps resemble at least those of the nearby cluster-forming clumps where only low-mass and intermediate-mass stars are forming (e.g., NGC 1333).

The central clump already harbors a cluster of protostars, whereas the northern and southern clumps show no active star formation. Since the northern clump has a mass and radius comparable to the central clump, the northern clump is a promissing candidate of pre-protocluster clump.

\subsection{Scenario of Clump Formation and Evolution}

We propose the following scenario of the clump formation and evolution in Serpens South. Figure 11] shows the conceptual diagram of the scenario.

(a) Fragmentation phase

Dense clumps form by fragmentation of a filamentary cloud that was close to magnetically critical. The fragmentation is likely to be driven by the ambipolar diffusion. In the absence of the ambipolar diffusion, the magnetically-critical filaments cannot fragment into clumps. Without the ambipolar diffusion, even in the mildly-magnetically-supercritical filaments, it is difficult to obtain the clumps whose separations are several times as large as the filament diameter, as seen in the dust continuum image, because the clump separations would become much larger. 
(b) Pre-protocluster phase

The clumps grow in mass by gas accumulation either along or across the magnetic field lines, or both. The merging of the fragments along the filament axis may also play a role in increasing the clump mass. This stage corresponds to the one immediately before active cluster formation is initiated. Thus, we refer to this stage as the pre-protocluster stage. The pre-protocluster clumps are the magnetically-supported clumps of cold, quiescent, dense gas.

(c) Early protocluster phase

When the density becomes high enough, active cluster formation is initiated and protostellar outflows start injecting supersonic turbulence in the clumps. Hence, the line widths of the clumps become larger.

(d) Quasi-virial equilibrium phase

Eventually, the clumps reach quasi-virial equilibrium due to the supersonic turbulence driven by protostellar outflows (see also Sugitani et al. 2010).

The southern, northern, and central clumps are presumably in Stages (a), (b) and (c), respectively.

We note that the physical properties of the pre-protocluster clumps identified in Serpens South are different from those expected from the accretion-driven turbulence scenario (Klessen \& Hennebelle 2010; Vazquez-Semadeni et al. 2010), for which the supersonic turbulence is maintained by the accretion flows onto the dense clumps. The accretion-driven turbulence scenario considers that star formation proceeds dynamically, and predicts that the turbulent energy balances with the gravitational energy in the dense clumps in formation. In contrast, the dense clumps in Serpens South appear more quiescent, in particular prior to the cluster formation. In Serpens South, stellar feedback is likely to be more responsible for driving turbulent motions in the dense clumps.

On the other hand, it remains unclear how the main filament was formed. The collision between the multiple filaments may have played a role because in the channel maps, several velocity-coherent components can be recognized. Further investigation is needed to understand how the Serpens South filament was formed.

\section{Physical Properties of Dense Cores}

As shown in Section 3, the $\mathrm{N}_{2} \mathrm{H}^{+}$velocity channel maps show that the clumps contain substructures, or dense cores. Here, we identify the dense cores from the $\mathrm{N}_{2} \mathrm{H}^{+}$data cube and estimate the physical properties of the dense cores.

We note that the core identification depends strongly on the angular and velocity resolutions. Thus, the identified cores may not be the actual units that are separated dynamically from the 
background media. However, we attempt to identify the substructures recognized in the current data as dense cores, following the previous studies.

\subsection{Identification of Dense Cores}

We apply the clumpfind to identify dense cores from the $\mathrm{N}_{2} \mathrm{H}^{+}$data cube Williams et al. 1994). The conditions adopted for the core identification are essentially the same as described in Ikeda et al. (2007). The threshold and stepsize of the core identification are set to the $2 \sigma$ noise level. A core identified must contain more than two continuous velocity channels and each channel must contain more than 3 pixels whose intensities are larger than the $3 \sigma$ noise levels. In addition, the core must have pixels connected to both the velocity and space domains (see Ikeda et al. 2007 for further details). Most of the $\mathrm{N}_{2} \mathrm{H}^{+}(J=1-0)$ hyperfine components are blended significantly over much of the area observed. Therefore, we used only the isolated component $\left(F_{1} F \rightarrow F_{1}^{\prime} F^{\prime}=01 \rightarrow 12\right)$ to identify the cores. A minimum mass of the core identified by this procedure is evaluated to about $0.17 \mathrm{M}_{\odot}$ (corresponding to about $60 \sigma$ levels) for $T_{\mathrm{ex}} \approx 7 \mathrm{~K}$ and $X_{\mathrm{N}_{2} \mathrm{H}^{+}} \approx 2.5 \times 10^{-10}$. In total, we identify 70 dense cores.

\subsection{Derivation of Physical Quantities}

We determine the physical properties of the cores are determined by using the definitions described in Section 3.3 of Ikeda et al. (2007). The position and local standard of rest (LSR) velocity of a core are set to the values at the pixel having the largest antenna temperature, $T_{\mathrm{A}, \text { peak }}^{*}$. The core radius, $R_{\text {core }}$, is calculated as the radius of a circle having the same area projected on the position-position plane as the identified core, by using Equation 2 of Ikeda et al. (2007). The aspect ratio of the core is calculated by the two-dimensional Gaussian fitting to its total integrated intensity distribution. The FWHM line width, $d V_{\text {core }}$, is corrected for the velocity resolution of the spectrometers $\left(=0.025 \mathrm{~km} \mathrm{~s}^{-1}\right)$. The Local Thermodynamic Equilibrium (LTE) mass is estimated under the assumption that all the hyperfine components are optically thin (see Ikeda et al. 2007 for further details). As mentioned in the previous section, the optically-thin assumption is reasonable in most of the area because the mean total optical depth derived from the hyperfine fitting is $\tau \sim 4-6$ except for the northern part. For simplicity, the $\mathrm{N}_{2} \mathrm{H}^{+}$fractional abundance relative to $\mathrm{H}_{2}$ is assumed to be spatially constant and equal to the mean fractional abundance of the observed area, $2.5 \times 10^{-10}$. The excitation temperatures are also set to be spatially constant at 4,7 , and 6 $\mathrm{K}$ (the mean values listed in Table 1) for the northern, central, and southern regions, respectively.

We note that the physical properties of the identified cores are partly affected by the core identification method and its adopted parameters (see Section 4.1 of Maruta et al. (2010) for further discussion). 


\subsection{Core Properties}

In Table 4, we show the physical quantities of the 70 dense cores identified from the $\mathrm{N}_{2} \mathrm{H}^{+}$data cube. In Table 5, we also list the minimum, maximum, and mean values of $R_{\text {core }}, d v_{\text {core }}, M_{\text {LTE }}$, $M_{\mathrm{vir}} / M_{\mathrm{LTE}}, \bar{n}$, and aspect ratio. Here, we compute these values by dividing the observed area into the three parts as in Table 1; north, center, and south.

Figures 12 through 12 indicate the histograms of the radius $\left(R_{\text {core }}\right)$, LTE mass $\left(M_{\mathrm{LTE}}\right)$, mean density $(\bar{n})$, line width $\left(\Delta V_{\text {core }}\right)$, virial ratio $\left(\alpha_{\text {vir }}\right)$, and aspect ratio of the $\mathrm{N}_{2} \mathrm{H}^{+}$cores. In Figures 12 through 12, the cyan, grey, and magenta histograms show the cores located in the northern, central, and southern areas, respectively. The distribution of the core radius takes its maximum at about $0.06 \mathrm{pc}$, ranging from 0.04 to $0.11 \mathrm{pc}$. The peak of its distribution is close to the mean of $0.065 \mathrm{pc}$ (see Figure $12 \mathrm{a}$ ). The maximum and minimum radii of the $\mathrm{N}_{2} \mathrm{H}^{+}$cores are very close to the mean core radius, indicating that the distribution of the core radius is very narrow. The cores in the southern part tend to be somewhat smaller than those in the other parts. On the other hand, the histogram of the LTE mass shows a much broader distribution than that of the core radius. The distribution of the LTE mass takes its maximum at about $4 \mathrm{M}_{\odot}$ ranging from 0.8 to $40 \mathrm{M}_{\odot}$ (see Figure $12 \mathrm{~b}$ ). The distribution of the mean density of the $\mathrm{N}_{2} \mathrm{H}^{+}$cores has a single peak at about $1 \times 10^{5} \mathrm{~cm}^{-3}$ that is close to the mean value (see Figure 12 $\mathrm{c}$ ). The distribution of the core mean density is also broad, ranging from $0.25 \times 10^{5}$ to $3.2 \times 10^{5} \mathrm{~cm}^{-3}$ (see Figure 12k). The cores located in the northern part tend to have larger LTE masses and larger mean densities, compared to the cores in the other parts.

Figure $12 \mathrm{~d}$ shows the distribution of the FWHM line width of the $\mathrm{N}_{2} \mathrm{H}^{+}$cores. The distribution of the line width has a single peak at around a mean of $0.45 \mathrm{~km} \mathrm{~s}^{-1}$, ranging from 0.078 to 0.85 $\mathrm{km} \mathrm{s}^{-1}$. Its distribution also appears to be concentrated in the range of 0.3 to $0.5 \mathrm{~km} \mathrm{~s}^{-1}$. Some cores in the central part have large line widths. Applying the procedure described by Myers et al. (1991), we classify the identified cores into the following two groups: gthermal corehand gturbulent core, h on the basis of the critical line width, $d V_{\mathrm{cr}}$. The critical line width is defined as follows:

$$
d V_{\text {cr }}=\left[8 \ln 2 k_{B} T\left(\frac{1}{\mu m_{H}}+\frac{1}{m_{\mathrm{obs}}}\right)\right]^{1 / 2},
$$

where $k_{B}$ is the Boltzmann constant, $\mu=2.33$ is the mean molecular weight, $m_{H}$ is the mass of a hydrogen atom, and $m_{\mathrm{obs}}$ is the mass of a observed molecule, i.e., $\mathrm{N}_{2} \mathrm{H}^{+}$in this paper. The critical line width of the $\mathrm{N}_{2} \mathrm{H}^{+}$cores is evaluated to be $0.55 \mathrm{~km} \mathrm{~s}^{-1}$ when the gas temperature of $T=14$ $\mathrm{K}$ is adopted. We note that $T=14 \mathrm{~K}$ is close to the dust temperature obtained from the SED fitting of the Herschel data. Among the 70 identified $\mathrm{N}_{2} \mathrm{H}^{+}$cores, most of the cores (about $76 \%$ ) are categorized as the thermal cores. Even for the cores classified as the turbulent cores, most of them have transonic nonthermal motions. In other words, the cores in the Serpens South IRDC are relatively quiescent.

Figure 12 e shows the distribution of the virial ratios. First, most of the cores have the virial

ratios not so far from unity. In addition, the virial ratios appear to depend weakly on area. For 
example, in the northern part, almost all the cores have the virial ratios smaller than unity. In contrast, in the central and southern parts, the majority of the cores have the virial ratios larger than unity.

Figure 12f shows the distribution of the aspect ratios of the cores. The majority of the cores have the aspect ratios close to unity. The cores in the southern part tend to be somewhat more elongated than those in the other parts.

\subsubsection{Line Width-Radius Relation}

Figure 13 a shows th line-width-radius relation of the 70 dense cores identified from the $\mathrm{N}_{2} \mathrm{H}^{+}$ data cube. For comparison, we show in Figure 13a the best-fit power-law that is given by

$$
\log \left(\frac{\Delta V_{\text {core }}}{\mathrm{km} \mathrm{s}^{-1}}\right)=(0.335 \pm 0.194)+(0.587 \pm 0.161) \log \left(\frac{R_{\text {core }}}{\mathrm{pc}}\right),
$$

where the correlation coefficient is $\mathcal{R}=0.40$. The line-width-radius relation of the identified cores does not show a clear power-law like the so-called Larson (1981)'s law. The weak dependence on the line widths of the core radius is also pointed out by recent studies of other star-forming regions such as in Orion A (Ikeda et al. 2007), $\rho$ Oph (Maruta et al. 2010), and massive-star-forming regions (Sanchez-Monge et al. 2013). The weak dependence of the line-width-radius relation may suggest that the identified cores are in various dynamical states as discussed below.

Figure $13 \mathrm{a}$ indicates that the nonthermal components of the line widths appear to be important in the identified cores. To quantify the contribution of the nonthermal components in the line widths of the cores, we plot in Figure $13 \mathrm{~b}$ the nonthermal line width $\Delta V_{\mathrm{NT}}\left[\equiv\left(\Delta V_{\text {core }}^{2}-8 \ln 2 k_{B} T / m_{\mathrm{obs}}\right)^{1 / 2}\right]$ as a function of core radius. For comparison, we also plot in Figure 13b the best-fit power-law that is given by

$$
\log \left(\frac{\Delta V_{\mathrm{NT}}}{\mathrm{km} \mathrm{s}^{-1}}\right)=(0.037 \pm 0.150)+(0.315 \pm 0.125) \log \left(\frac{R_{\text {core }}}{\mathrm{pc}}\right)
$$

with $\mathcal{R}=0.36$. Again, the correlation between the nonthermal line width and core radius is weak.

The weak dependence of the line width on the core radius is consistent with the results of the recent numerical simulations of turbulent, magnetized molecular clouds. The numerical simulations show that the cores formed in turbulent media are not in perfect virial equilibrium, but in various dynamical states that are influenced by the ambient environments (Nakamura \& Li 2008, 2011; Offner et al. 2008). 


\subsubsection{Mass-Radius Relation}

In Figure 13k, we present the LTE mass as a function of core radius. For comparison, we plot with a solid line the best-fit power-law that is given by

$$
\log \left(\frac{M_{\mathrm{LTE}}}{M_{\odot}}\right)=(3.32 \pm 0.35)+(2.17 \pm 0.29) \log \left(\frac{R_{\text {core }}}{\mathrm{pc}}\right)
$$

where $\mathcal{R}=0.69$. The relation of $M_{\mathrm{LTE}}=4 \pi \mu m_{H} \bar{n} R_{\text {core }}^{3} / 3$ is also shown with a dashed line in Figure 13k, where the mean density is set to $\bar{n}=9.6 \times 10^{4} \mathrm{~cm}^{-3}$, which is close to the critical density of $\mathrm{N}_{2} \mathrm{H}^{+}(J=1-0), n_{\mathrm{cr}} \simeq 1.5 \times 10^{5} \mathrm{~cm}^{-3}$.

\subsubsection{Virial Ratio-LTE-Mass Relation}

The importance of the self-gravity in a core is often assessed using the virial ratio, the ratio between the virial mass and core mass $\left(\alpha_{\mathrm{vir}}=M_{\mathrm{vir}} / M_{\mathrm{LTE}}\right)$. In Figure 13d, we present the virial ratio as a function of the LTE mass, where we adopt a dimensionless parameter of $a=1$, corresponding to a uniform sphere. For comparison, we show in Figure 13 d the best-fit power-law function that is given by

$$
\log \alpha_{\text {vir }}=(0.53 \pm 0.04)+(-0.651 \pm 0.050) \log \left(\frac{M_{\mathrm{LTE}}}{M_{\odot}}\right),
$$

with the correlation coefficient of $\mathcal{R}=0.85$. The virial ratio ranges from 0.3 to 3.5 , having a mean of 1.4. Figure $13 \mathrm{~d}$ indicates that the cores with larger LTE masses tend to have smaller virial ratios. The majority of the cores have virial ratios smaller than 2, indicating that the total energy (the gravitational plus internal kinetic energies) of a core is negative. Therefore, the self-gravity of the individual cores appears to be important for the majority of the cores.

However, we note that the physical properties of the cores identified by the above procedure (and any other core identification schemes) depend strongly on the telescope beam size because the structures smaller than the beam size cannot be spatially resolved (see Maruta et al. 2010). According to Maruta et al. (2010), the cores identified from the data having the higher spatial resolution tend to be gravitational unbound and the external pressure due to the ambient turbulence plays an important role in the formation and evolution of the cores (see also Figure 10 of Nakamura \& Li 2011). It remains uncertain whether a core identified here is a basic structure that is separated dynamically from the ambient turbulent media. In fact, recently, Nakamura et al. (2012) revealed that the two starless cores which was previously identified as single cores using the single-dish telescopes, contain several substellar-mass condensations whose masses are larger than or at least comparable to the critical Bonner-Ebert mass. The substellar-mass condensations might be real basic units of star formation in molecular clouds. The data with higher angular resolution on the basis of the interferometric observations will be necessary to address the issue of what is the basic units of star formation in star-forming molecular clouds. 


\section{Conclusion}

We summarize the main results of the present paper as follows.

1. From the Herschel and $1.1 \mathrm{~mm}$ dust continuum data, we identified three dense clumps along the main filament: northern (V-shap), central (cluster-forming), and southern clumps. The clump masses are estimated to be around $40-230 M_{\odot}$.

2. Applying a hyperfine fitting to the $\mathrm{N}_{2} \mathrm{H}^{+}(J=1-0)$ data, we derived the spatial distributions of the $\mathrm{N}_{2} \mathrm{H}^{+}$column density, excitation temperature, fractional abundance, optical depth, and line width in Serpens South. We found that the cluster-forming clump has larger line widths of about $1 \mathrm{~km} \mathrm{~s}^{-1}$, whereas the regions with no signs of active star formation have smaller line widths of about $0.6 \mathrm{~km} \mathrm{~s}^{-1}$. The fractional abundance of $\mathrm{N}_{2} \mathrm{H}^{+}$is estimated to be $X_{\mathrm{N}_{2} \mathrm{H}^{+}} \simeq 2.5 \times 10^{-10}$ over the whole area and tends to decrease with increasing column density. We interpret that the overall dependence of $X_{\mathrm{N}_{2} \mathrm{H}^{+}}$on the column density reflects the ionization degree in the dense molecular gas, which is determined by the balance between the cosmic-ray ionization and the recombination.

3. Applying the virial analysis, we found that all the three clumps have small virial ratios of $0.1-0.3$. This indicates that the internal turbulent motions play only a minor role in the clump support. The northern clump has a mass and radius comparable to the central cluster-forming clump, although there is no sign of active cluster formation. Thus, it is a likely candidate of pre-protocluster clump, where active cluster formation will be in the future.

4. Although the clumps show the observational signs of the global infall motions, the infall speed appears to be much slower than the free-fall velocities of a few $\mathrm{km} \mathrm{s}^{-1}$. We propose that the slow global infall is due to the fact that the Serpens South filament is supported by the large-scale ordered magnetic field that was discovered by Sugitani et al. (2011). According to our estimation, the parent cloud was magnetically critical and either the ambipolar diffusion or mass accretion along the magnetic field lines may have triggerd the global infall and the active cluster formation that is observed in the central clump.

5. The physical properties of the pre-protocluster clump are (1) the column density higher than $10^{23} \mathrm{~cm}^{-2}$, (2) a lower temperature compared to the ambient gas, (3) a small internal velocity dispersion, (4) a small virial ratio, and (5) the magnetic field that is close to the critical. These properties contradict the accretion-driven turbulence scenario, which predicts that in the pre-protocluster clump, the turbulent energy should balance with the gravitational energy due to the momentum injection driven by the global accretion flow.

6. Applying the clumpfind to the $3 \mathrm{D}$ data cube of the $\mathrm{N}_{2} \mathrm{H}^{+}$emission taken with the Nobeyama 45-m telescope, we identified 70 dense cores. The cores with larger LTE masses tend to have smaller virial ratios. The majority of the cores have virial ratios smaller than 2 , indicating that for most of the cores, the total energy (the gravitational plus internal kinetic energies) is negative. Therefore, the self-gravity appears to be important for most of the cores. A caveat is that the physical properties of the identified cores depend strongly on the telescope beam size because the 
substructures smaller than the telescope beam size $\left(\approx 20^{\prime \prime}\right.$, corresponding to $\left.0.04 \mathrm{pc}\right)$ cannot be resolved. Higher spatial resolution and higher sensitivity observations will be necessary to uncover the basic units of star formation.

This work is supported in part by a Grant-in-Aid for Scientific Research of Japan (20540228). We thank Thushara Pillai, Jens Kauffmann, Alvaro Hacar, Philippe André, and Huei-Ru Chen for valuable comments and suggestions. We are grateful to the staffs at the Nobeyama Radio Observatory (NRO) for both operating the 45-m and helping us with the data reduction. NRO is a branch of the National Astronomical Observatory, National Institutes of Natural Sciences, Japan. We also thank the staffs at the Caltech Submillimeter Observatory for giving us an opportunity to use the telescope.

\section{REFERENCES}

André, P., Menshchikov, A., Bontemps, S., et al. 2010, A\&A, 518, L102

André, P., Ward-Thompson, D., \& Barsony, M. 2000, in Protostars and Planets IV, Tucson: University of Arizona Press, eds. Mannings, V., Boss, A. P., \& Russell, S. S. p. 59

Arzoumanian, D., André, P., Peretto, N., \& Könyves, V. 2013, A\&A, 553, 119

Bailey, N. D. \& Basu, S. 2012, ApJ, 761, 67

Bertoldi, F. \& McKee, C. F. 1992, ApJ, 395, 140

Bontemps, S., André, P., Könyves, V., et al. 2010, A\&A, 518, L85

Busquet, G., Zhang, Q., Palau, A., et al. 2013, ApJ, 764, 26

Caselli, P., Benson, P. J., Myers, P. C., \& Tafalla, M. 2002, ApJ, 572, 238

Caselli, P., Myers, P. C., \& Thaddeus, P. 1995, ApJ, 455, L77

Di Francesco, J., André, P., \& Myers, P. C., 2004, ApJ, 617, 425

Dzib, S., Loinard, L., Mioduszewski, A. J., et al. 2010, ApJ, 718, 610

Egan, M. P., Shipman, R. F., Price, S. D., et al. 1998, ApJ, L199

Evans, N. J. II, Dunham, M. M., Jorgensen, J. K., et al. 2009, ApJS, 181, 321

Elmegreen, B. G. 1979, ApJ, 232, 729

Enoch, M. L., Young, K. E., Glenn, J., et al. 2006, ApJ, 638, 293

Fiege, J. D. \& Pudritz, R. E. 2000, MNRAS, 311, 105 
Friesen, R. K., Di Francesco, J., Shimajiri, Y., \& Takakuwa, S. 2010, ApJ, 708, 1002

Fuller, G. A., \& Myers, P. C. 1992, ApJ, 384, 523

Gutermuth, R. A., Bourke, T. L., Allen, L. E., et al. ApJ, 2008, 673, L151

Hacar, A., Tafalla, M., Kauffmann, J., Kovacs, A. 2013, A\&A, in press (arXiv:1303.2118)

Hernandez, A. K., \& Tan, J. C. 2012, ApJ, 730, 44

Heyer, M. H., \& Brunt, C. M. 2004, ApJ, 615, L45

Ikeda, N., Sunada, K., \& Kitamura, Y. 2007, ApJ, 665, 1194

Kauffmann, J. \& Pillai, T. 2010, ApJ, 723, 7

Kauffmann, J., Pillai, T., Shetty, R., Myers, P. C., \& Goodman, A. A. 2010, ApJ, 716, 433

Kirk, H., Myers, P. C., Bourke, T. L., et al. 2013, ApJ, 766, 115

Klessen, R. S., \& Hennebelle, P., 2010, A\&A, 520, 17

Könyves, V., André, P., Menshchikov, A., et al. 2010, A\&A, 518, L106

Krumholz, M. R. \& McKee, C. F. 2008, Nature, 451, 28

Kutner, M. L., \& Ulich, B. L. 1981, ApJ, 250, 341

Larson, R. B. 1981, MNRAS, 194, 809

Li, D., Kauffmann, J., Zhang, Q., \& Chen, W. 2012, submitted to ApJL (arXiv:1207.1178)

Liu, G., Calzetti, D., Yun, M. S., et al. 2010, AJ, 139, 1190

Maruta, H., Nakamura, F., Nishi, R., et al. 2010, ApJ, 714, 680

Matsumoto, T., Nakamura, F., \& Hanawa, T. 1994, PASJ, 46, 243

Maury, A., P. André, Men'shchikov, A., Konyves, V., \& Bontemps, S. 2011, A\&A, 535, 77

Miettinen, O. 2012, A\&A, 540, 104

Myers, P. C. 2009, ApJ, 700, 1609

Myers, P. C., Ladd, E. F., \& Fuller, G. A. 1991, ApJ, 372, L95

Myers, P. C., Mardones, D., Tafalla, M., Williams, J. P., \& Wilner, D. J. 1996, ApJ, 465, 133

Nakamura, F. \& Li, Z.-Y. 2008, ApJ, 687, 354 
Nakamura, F., \& Li, Z.-Y. 2010, in IAU Symp. 270, Computational Star Formation, ed. J. Alves et al. (Cambridge: Cambridge Univ. Press), 115

Nakamura, F. \& Li, Z.-Y. 2011, ApJ, 740, 36

Nakamura, F., Hanawa, T., \& Nakano, T. 1993, PASJ, 45, 551

Nakamura, F., Sugitani, K., Shimajiri, Y. et al. 2011, ApJ, 737, 56

Nakamura, F., Takakuwa, S., \& Kawabe, R. 2012, ApJ, 758, 25

Nakano, T. 1998, ApJ, 494, 587

Offner, S. S. R., Klein, R. I., \& McKee, C. F. 2008, ApJ, 686, 1174

Ostriker, J. 1964, ApJ, 140, 1056

Perault, M., Omont, A., Simon, G., et al. 1996, A\&A, 315, 165

Peretto, N., \& Fuller, G. A. 2009, A\&A, 505, 405

Pillai, T., Kauffmann, J., Wyrowski, F., et al. A\&A, 530, 118

Pillai, T., Wyrowski, F., Carey, S. J., \& Menten, K. M. 2006, A\&A, 450, 569

Plume, R., Jaffe, D. T., \& Evaqns, N. J., II. 1997, ApJ, 476, 730

Rathborne, J. M., Jackson, J. M., \& Simon, R. 2006, ApJ, 641, 389

Rygl, K. L. J., Wyrowski, F., Schuller, F., \& Menten, K. M. 2010, A\&A, 515, 42

Saito, H., Saito, M., Yonekura, Y., \& Nakamura, F. 2008, ApJS, 178, 302

Sanchez-Monge, A., Palau, A., Fontani, F., et al. 2013, MNRAS, 432, 3288

Sawada, T., Ikeda, N., Sunada, K., et al. 2008, PASJ, 60, 445

Schneider, N., Csengeri, T., Bontemps, S., et al. 2010, A\&A, 520, 49

Shimajiri, Y., Kawabe, R., Takakuwa, S., et al. 2011, PASJ, 63, 105

Sorai, K., Sunada, K., Okumura, S. K., et al. 2000, Proc. SPIE, 4015, 86

Stodółkiewicz, J. S. 1963, Acta Astron. 13, 30

Sugitani, K., Nakamura, F., Tamura, M., et al. 2010, ApJ, 716, 299

Sugitani, K., Nakamura, F., Watanabe, M., et al. 2011, ApJ, 734, 63

Sunada, K., Yamaguchi, C., Nakai, N., et al. 2000, Proc. SPIE, 4015, 237 
Teixeira, G. D. C., Kumar, M. S. N., Bachiller, R., \& Grave, M. C. 2012, A\&A, 543, 140

Tiné, S., Roueff, E., Falgarone, E., et al. 2000,A\&A, 356, 1039

Yamaguchi, C., Sunada, K., Iizuka, Y., et al. 2000, Proc. SPIE, 4015, 614

Vazquez-Semadeni, E., Colin, P., Gomez, G. C., et al. 2010, ApJ, 715, 1302

Walsh, A. J., Myers, P. C., Di Francesco, J., et al. 2007, ApJ, 655, 958

Williams, J. P., de Geus, E. J., \& Blitz, L. 1994, ApJ, 428, 693 
Table 1. $\mathrm{N}_{2} \mathrm{H}^{+}$Line Characteristics in Serpens South

\begin{tabular}{lllll}
\hline \hline Physical Quantities & \multicolumn{1}{c}{ Mean $^{\mathrm{a}}$} & $\sigma^{\mathrm{b}}$ & \multicolumn{1}{c}{ Min $^{\mathrm{a}}$} & \multicolumn{1}{c}{ Max $^{\mathrm{a}}$} \\
\hline whole area & & & & \\
\hline$V_{\mathrm{LSR}}\left(\mathrm{km} \mathrm{s}^{-1}\right)$ & $7.34 \pm 0.06$ & 0.31 & $6.70 \pm 0.06$ & $7.76 \pm 0.07$ \\
$\Delta V\left(\mathrm{~km} \mathrm{~s}^{-1}\right)$ & $0.81 \pm 0.08$ & 0.33 & $0.32 \pm 0.08$ & $1.48 \pm 0.06$ \\
$T_{\text {ex }}(\mathrm{K})$ & $6.0 \pm 1.6$ & 2.3 & $3.6 \pm 0.84$ & $18.6 \pm 5.75$ \\
$\tau_{\text {tot }}$ & $7.6 \pm 2.9$ & 6.4 & $0.5 \pm 0.18$ & $34.4 \pm 10.2$ \\
\hline north & & & & \\
\hline$V_{\mathrm{LSR}}\left(\mathrm{km} \mathrm{s}^{-1}\right)$ & $7.59 \pm 0.06$ & 0.09 & $7.29 \pm 0.06$ & $7.76 \pm 0.07$ \\
$\Delta V\left(\mathrm{~km} \mathrm{~s}^{-1}\right)$ & $0.65 \pm 0.08$ & 0.11 & $0.44 \pm 0.10$ & $0.96 \pm 0.10$ \\
$T_{\text {ex }}(\mathrm{K})$ & $4.1 \pm 0.89$ & 0.2 & $3.6 \pm 0.84$ & $4.6 \pm 1.05$ \\
$\tau_{\text {tot }}$ & $14.4 \pm 5.31$ & 7.4 & $5.4 \pm 1.49$ & $34.4 \pm 10.2$ \\
\hline center & & & & \\
\hline$V_{\mathrm{LSR}}\left(\mathrm{km} \mathrm{s}^{-1}\right)$ & $7.37 \pm 0.06$ & 0.16 & $7.00 \pm 0.06$ & $7.66 \pm 0.06$ \\
$\Delta V\left(\mathrm{~km} \mathrm{~s}^{-1}\right)$ & $1.05 \pm 0.08$ & 0.35 & $0.32 \pm 0.08$ & $1.48 \pm 0.06$ \\
$T_{\text {ex }}(\mathrm{K})$ & $7.4 \pm 1.95$ & 2.5 & $4.1 \pm 0.89$ & $18.6 \pm 5.75$ \\
$\tau_{\text {tot }}$ & $3.8 \pm 1.37$ & 1.9 & $0.5 \pm 0.18$ & $9.6 \pm 3.49$ \\
\hline south & & & & \\
\hline$V_{\mathrm{LSR}}\left(\mathrm{km} \mathrm{s}^{-1}\right)$ & $6.95 \pm 0.06$ & 0.30 & $6.70 \pm 0.06$ & $7.63 \pm 0.08$ \\
$\Delta V\left(\mathrm{~km} \mathrm{~s}^{-1}\right)$ & $0.59 \pm 0.08$ & 0.16 & $0.37 \pm 0.07$ & $1.07 \pm 0.09$ \\
$T_{\text {ex }}(\mathrm{K})$ & $5.7 \pm 1.78$ & 1.6 & $4.0 \pm 1.03$ & $17.3 \pm 20.8$ \\
$\tau_{\text {tot }}$ & $6.0 \pm 2.51$ & 2.3 & $0.6 \pm 0.87$ & $10.7 \pm 7.03$
\end{tabular}

${ }^{a}$ With standard deviation of the error of the hyperfine fitting including the flux calibration error of about $\pm 20 \%$ and the spectrometer's velocity resolution of $0.05 \mathrm{~km} \mathrm{~s}^{-1}$

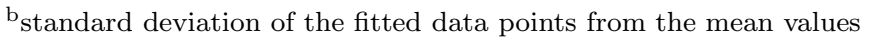

Note. - The whole observed area is divided into three subregions: (1) north (Decl. $\geq-2002.4$ ), (2) center (-2 $0532.5 \leq$ Decl. $\leq-2002.4$ ), and (3) south (Decl. $\leq-20532.5$ ). 
Table 2. Column Densities and $\mathrm{N}_{2} \mathrm{H}^{+}$Fractional Abundances in Serpens South

\begin{tabular}{lcccc}
\hline \hline Physical Quantities & Mean $^{\mathrm{a}}$ & $\sigma^{\mathrm{b}}$ & Min $^{\mathrm{a}}$ & Max $^{\mathrm{a}}$ \\
\hline whole area & & & & \\
\hline$N_{\mathrm{H}_{2}}\left(10^{22} \mathrm{~cm}^{-2}\right)$ & $6.9 \pm 1.4$ & 1.5 & $1.5 \pm 0.3$ & $20.1 \pm 4.0$ \\
$N_{\mathrm{N}_{2} \mathrm{H}^{+}}\left(10^{13} \mathrm{~cm}^{-2}\right)$ & $1.7 \pm 0.5$ & 0.8 & $0.4 \pm 0.1$ & $4.0 \pm 1.0$ \\
$X_{\mathrm{N}_{2} \mathrm{H}}\left(10^{-10}\right)$ & $2.5 \pm 1.4$ & 0.7 & $1.1 \pm 0.4$ & $6.3 \pm 4.2$ \\
\hline north & & & & \\
\hline$N_{\mathrm{H}_{2}}\left(10^{22} \mathrm{~cm}^{-2}\right)$ & $8.3 \pm 1.7$ & 1.2 & $2.4 \pm 0.5$ & $14.2 \pm 2.8$ \\
$N_{\mathrm{N}_{2} \mathrm{H}}+\left(10^{13} \mathrm{~cm}^{-2}\right)$ & $2.0 \pm 0.6$ & 0.8 & $0.8 \pm 0.4$ & $4.0 \pm 1.0$ \\
$X_{\mathrm{N}_{2} \mathrm{H}}+\left(10^{-10}\right)$ & $2.5 \pm 1.1$ & 0.7 & $1.5 \pm 0.6$ & $6.3 \pm 4.2$ \\
\hline center & & & & \\
\hline$N_{\mathrm{H}_{2}}\left(10^{22} \mathrm{~cm}^{-2}\right)$ & $7.2 \pm 1.4$ & 1.7 & $1.5 \pm 0.3$ & $20.1 \pm 4.0$ \\
$N_{\mathrm{N}_{2} \mathrm{H}^{+}}\left(10^{13} \mathrm{~cm}^{-2}\right)$ & $1.6 \pm 0.5$ & 0.7 & $0.5 \pm 0.2$ & $3.5 \pm 0.7$ \\
$X_{\mathrm{N}_{2} \mathrm{H}}\left(10^{-10}\right)$ & $2.6 \pm 1.7$ & 0.8 & $1.1 \pm 0.4$ & $4.9 \pm 2.0$ \\
\hline south & & & & \\
\hline$N_{\mathrm{H}_{2}}\left(10^{22} \mathrm{~cm}^{-2}\right)$ & $4.8 \pm 1.0$ & 0.5 & $2.4 \pm 0.5$ & $7.4 \pm 1.5$ \\
$N_{\mathrm{N}_{2} \mathrm{H}}\left(10^{13} \mathrm{~cm}^{-2}\right)$ & $1.2 \pm 0.5$ & 0.4 & $0.4 \pm 0.1$ & $2.0 \pm 0.5$ \\
$X_{\mathrm{N}_{2} \mathrm{H}}+\left(10^{-10}\right)$ & $2.4 \pm 1.5$ & 0.5 & $1.1 \pm 0.4$ & $3.6 \pm 1.6$ \\
\hline
\end{tabular}

Note. - The definitions of the subareas are the same as those of Table 1

${ }^{a}$ With standard deviation of the error of the hyperfine fitting including the flux calibration error of about $\pm 20 \%$ and the spectrometer's velocity resolution of $0.05 \mathrm{~km} \mathrm{~s}^{-1}$

${ }^{\mathrm{b}}$ standard deviation of the fitted data points from the mean values

Table 3. Physical Quantities of Clumps in Serpens South

\begin{tabular}{llll}
\hline \hline Physical Quantities & Serps N & Serps C & Serps S \\
\hline Mass $\left(M_{\odot}\right)$ & 193 & 232 & 36 \\
Radius $(\mathrm{pc})$ & 0.189 & 0.200 & 0.093 \\
$T_{\text {dust }}(K)^{a}$ & 11.3 & 14.0 & 12.7 \\
$\Delta V\left(\mathrm{~km} \mathrm{~s}^{-1}\right)$ & 0.665 & 1.25 & 0.580 \\
$U\left(M_{\odot} \mathrm{km}^{2} \mathrm{~s}^{-2}\right)$ & 36 & 114 & 5.7 \\
$W\left(M_{\odot} \mathrm{km}^{2} \mathrm{~s}^{-2}\right)$ & -721 & -1000 & -43.6 \\
$\alpha_{\text {vir }}$ & 0.10 & 0.23 & 0.26 \\
$\Gamma^{b}$ & 1.5 & 1.6 & 1.1 \\
\hline
\end{tabular}

${ }^{a}$ Column-density weighted dust temperature derved from the SED fitting of the Herschel data.

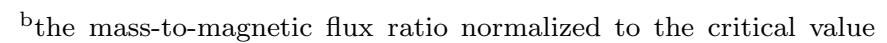
$2 \pi G^{1 / 2}$. 
Table 4. Properties of the $\mathrm{N}_{2} \mathrm{H}^{+}$cores in Serpens South

\begin{tabular}{|c|c|c|c|c|c|c|c|c|c|c|c|c|c|}
\hline ID & $\begin{array}{c}\text { R.A. } \\
(\mathrm{J} 2000.0)\end{array}$ & $\begin{array}{c}\text { Decl. } \\
(\mathrm{J} 2000.0)\end{array}$ & $\begin{array}{c}V_{\mathrm{LSR}} \\
\left(\mathrm{km} \mathrm{s}^{-1}\right)\end{array}$ & $\begin{array}{l}T_{A, \text { peak }}^{*}{ }^{\mathrm{a}} \\
(\mathrm{K})\end{array}$ & $\begin{array}{c}R_{\text {core }} \\
(\operatorname{arcsec})\end{array}$ & $\begin{array}{l}R_{\text {core }} \\
(\mathrm{pc})\end{array}$ & $\begin{array}{c}\text { Aspect } \\
\text { Ratio }\end{array}$ & $\begin{array}{c}\Delta v_{\text {core }} \\
\left(\mathrm{km} \mathrm{s}^{-1}\right)\end{array}$ & $\begin{array}{l}M_{\mathrm{LTE}} \\
\left(M_{\odot}\right)\end{array}$ & $\begin{array}{l}M_{\text {vir }} \\
\left(M_{\odot}\right)\end{array}$ & $\alpha_{\mathrm{vir}}$ & $\begin{array}{c}\bar{n} \\
\left(10^{5} \mathrm{~cm}^{-3}\right)\end{array}$ & Area $^{b}$ \\
\hline 1 & 182944.9 & -020533.0 & 8.87 & 0.65 & 27.4 & 0.0551 & 3.26 & 0.438 & 1.48 & 5.12 & 3.45 & 0.37 & $\mathrm{~s}$ \\
\hline 2 & 182947.1 & -020046.6 & 7.51 & 0.61 & 35.0 & 0.0705 & 1.12 & 0.312 & 2.09 & 5.16 & 2.47 & 0.25 & c \\
\hline 3 & 182949.5 & -02 0509.4 & 7.62 & 0.56 & 31.3 & 0.0629 & 1.05 & 0.324 & 2.01 & 4.70 & 2.33 & 0.34 & c \\
\hline 4 & 182949.6 & -015808.0 & 7.85 & 0.76 & 25.7 & 0.0518 & 1.29 & 0.370 & 6.19 & 4.22 & 0.68 & 1.84 & $\mathrm{n}$ \\
\hline 5 & 182952.3 & -015746.9 & 7.88 & 0.71 & 27.1 & 0.0545 & 1.41 & 0.331 & 5.12 & 4.12 & 0.80 & 1.31 & $\mathrm{n}$ \\
\hline 6 & 182954.2 & -020048.2 & 7.43 & 0.61 & 38.0 & 0.0764 & 1.03 & 0.677 & 4.50 & 11.34 & 2.52 & 0.42 & $\mathrm{c}$ \\
\hline 7 & 182955.2 & -015839.8 & 7.51 & 0.94 & 52.3 & 0.1052 & 1.22 & 0.342 & 23.62 & 8.12 & 0.34 & 0.84 & $\mathrm{n}$ \\
\hline 8 & 182955.7 & -01 5929.6 & 7.72 & 0.63 & 25.5 & 0.0513 & 1.47 & 0.364 & 5.47 & 4.13 & 0.75 & 1.68 & $\mathrm{n}$ \\
\hline 9 & 182955.9 & -015834.9 & 7.93 & 0.61 & 32.7 & 0.0658 & 1.23 & 0.275 & 5.90 & 4.51 & 0.76 & 0.86 & $\mathrm{n}$ \\
\hline 10 & 182956.4 & -02 0121.4 & 8.02 & 0.62 & 31.2 & 0.0628 & 1.27 & 0.396 & 4.34 & 5.38 & 1.24 & 0.72 & c \\
\hline 11 & 182956.6 & -015751.8 & 7.62 & 0.92 & 29.9 & 0.0601 & 1.77 & 0.502 & 16.53 & 6.34 & 0.38 & 3.15 & $\mathrm{n}$ \\
\hline 12 & 182956.8 & -015958.8 & 7.74 & 0.61 & 23.4 & 0.0471 & 1.45 & 0.374 & 4.59 & 3.86 & 0.84 & 1.82 & $\mathrm{n}$ \\
\hline 13 & 182957.5 & -01 5944.8 & 7.22 & 0.67 & 24.9 & 0.0500 & 1.45 & 0.389 & 5.02 & 4.23 & 0.84 & 1.66 & $\mathrm{n}$ \\
\hline 14 & 182957.6 & -020040.3 & 7.30 & 0.63 & 30.6 & 0.0615 & 1.18 & 0.727 & 6.85 & 10.04 & 1.47 & 1.22 & c \\
\hline 15 & 182957.8 & -020144.3 & 7.17 & 0.60 & 37.1 & 0.0746 & 1.81 & 0.849 & 4.78 & 15.16 & 3.17 & 0.48 & c \\
\hline 16 & 182958.3 & -02 $06 \quad 27.1$ & 7.71 & 0.61 & 33.8 & 0.0679 & 1.82 & 0.515 & 3.18 & 7.35 & 2.31 & 0.42 & $\mathrm{~s}$ \\
\hline 17 & 182958.6 & $-0201 \quad 15.2$ & 7.65 & 0.89 & 32.0 & 0.0644 & 1.24 & 0.391 & 7.35 & 5.46 & 0.74 & 1.14 & $\mathrm{c}$ \\
\hline 18 & 182958.8 & -020150.0 & 7.98 & 0.67 & 26.2 & 0.0528 & 2.45 & 0.308 & 2.12 & 3.84 & 1.81 & 0.60 & $\mathrm{c}$ \\
\hline 19 & 182958.8 & -015905.0 & 7.54 & 0.66 & 34.6 & 0.0695 & 1.01 & 0.503 & 12.21 & 7.34 & 0.60 & 1.50 & $\mathrm{n}$ \\
\hline 20 & 182959.5 & -02 0333.7 & 7.53 & 0.65 & 42.9 & 0.0864 & 1.28 & 0.768 & 6.48 & 15.21 & 2.35 & 0.42 & c \\
\hline 21 & 182959.5 & -015813.0 & 7.80 & 0.78 & 31.3 & 0.0630 & 1.05 & 0.644 & 10.28 & 8.79 & 0.86 & 1.70 & $\mathrm{n}$ \\
\hline 22 & 182959.6 & -020051.6 & 8.04 & 0.64 & 30.5 & 0.0614 & 1.16 & 0.330 & 2.90 & 4.64 & 1.60 & 0.52 & c \\
\hline 23 & 182960.0 & -020543.0 & 7.75 & 0.66 & 29.2 & 0.0588 & 1.46 & 0.399 & 2.91 & 5.06 & 1.74 & 0.59 & $\mathrm{~s}$ \\
\hline 24 & 183000.2 & -015955.6 & 7.37 & 0.91 & 29.6 & 0.0596 & 1.13 & 0.518 & 13.10 & 6.49 & 0.50 & 2.56 & $\mathrm{n}$ \\
\hline 25 & 183000.7 & $\begin{array}{lll}-02 & 02 & 19.9\end{array}$ & 7.04 & 0.97 & 47.7 & 0.0961 & 1.16 & 0.733 & 18.03 & 15.86 & 0.88 & 0.84 & c \\
\hline 26 & 183000.8 & $-0206 \quad 15.3$ & 7.43 & 0.65 & 22.7 & 0.0458 & 1.01 & 0.285 & 1.75 & 3.19 & 1.82 & 0.76 & $\mathrm{~s}$ \\
\hline 27 & 183001.0 & -02 0711.9 & 7.91 & 0.69 & 32.0 & 0.0644 & 1.12 & 0.533 & 2.74 & 7.23 & 2.64 & 0.42 & $\mathrm{~s}$ \\
\hline 28 & 183001.2 & -020122.6 & 7.96 & 0.91 & 19.9 & 0.0400 & 1.01 & 0.353 & 3.84 & 3.15 & 0.82 & 2.49 & c \\
\hline 29 & 183001.3 & -02 0421.3 & 7.70 & 0.59 & 38.3 & 0.0771 & 1.22 & 0.418 & 3.81 & 6.89 & 1.81 & 0.34 & c \\
\hline 30 & 183001.3 & -020418.0 & 6.54 & 0.66 & 38.7 & 0.0779 & 1.13 & 0.540 & 3.54 & 8.85 & 2.50 & 0.31 & $\mathrm{c}$ \\
\hline
\end{tabular}


Table 4-Continued

\begin{tabular}{|c|c|c|c|c|c|c|c|c|c|c|c|c|c|}
\hline ID & $\begin{array}{c}\text { R.A. } \\
\text { (J2000.0) }\end{array}$ & $\begin{array}{c}\text { Decl. } \\
(\mathrm{J} 2000.0)\end{array}$ & $\begin{array}{c}V_{\mathrm{LSR}} \\
\left(\mathrm{km} \mathrm{s}^{-1}\right)\end{array}$ & $\begin{array}{l}T_{A, \text { peak }}^{*}{ }^{\mathrm{a}} \\
(\mathrm{K})\end{array}$ & $\begin{array}{c}R_{\text {core }} \\
(\operatorname{arcsec})\end{array}$ & $\begin{array}{l}R_{\text {core }} \\
(\mathrm{pc})\end{array}$ & $\begin{array}{c}\text { Aspect } \\
\text { Ratio }\end{array}$ & $\begin{array}{c}\Delta v_{\text {core }} \\
\left(\mathrm{km} \mathrm{s}^{-1}\right)\end{array}$ & $\begin{array}{l}M_{\mathrm{LTE}} \\
\left(M_{\odot}\right)\end{array}$ & $\begin{array}{l}M_{\mathrm{vir}} \\
\left(M_{\odot}\right)\end{array}$ & $\alpha_{\mathrm{vir}}$ & $\begin{array}{c}\bar{n} \\
\left(10^{5} \mathrm{~cm}^{-3}\right)\end{array}$ & Area $^{b}$ \\
\hline 31 & 183001.5 & -02 0126.1 & 7.49 & 0.98 & 22.0 & 0.0444 & 1.38 & 0.338 & 3.39 & 3.40 & 1.00 & 1.61 & $\mathrm{c}$ \\
\hline 32 & 183001.6 & -02 0718.3 & 5.30 & 0.69 & 21.3 & 0.0428 & 2.02 & 0.078 & 0.79 & 2.31 & 2.93 & 0.42 & $\mathrm{~s}$ \\
\hline 33 & 183001.8 & -020529.5 & 7.87 & 0.70 & 36.1 & 0.0727 & 1.23 & 0.466 & 4.91 & 7.14 & 1.46 & 0.53 & $\mathrm{c}$ \\
\hline 34 & 183002.0 & -020100.6 & 7.04 & 0.95 & 38.4 & 0.0773 & 1.00 & 0.449 & 10.90 & 7.34 & 0.67 & 0.98 & $\mathrm{c}$ \\
\hline 35 & 183002.5 & $\begin{array}{lll}-02 & 02 & 12.9\end{array}$ & 7.90 & 1.04 & 42.2 & 0.0849 & 1.07 & 0.513 & 14.22 & 9.14 & 0.64 & 0.96 & c \\
\hline 36 & 183003.0 & -02 0301.2 & 7.22 & 1.65 & 49.0 & 0.0986 & 1.29 & 0.296 & 22.11 & 7.01 & 0.32 & 0.95 & $\mathrm{c}$ \\
\hline 37 & 183003.1 & -02 0307.4 & 6.75 & 1.21 & 43.9 & 0.0884 & 1.16 & 0.450 & 15.01 & 8.41 & 0.56 & 0.90 & $\mathrm{c}$ \\
\hline 38 & 183003.1 & -020441.6 & 7.07 & 1.39 & 46.6 & 0.0937 & 1.29 & 0.595 & 19.75 & 11.87 & 0.60 & 0.99 & $\mathrm{c}$ \\
\hline 39 & 183003.4 & -020315.0 & 7.71 & 1.48 & 54.1 & 0.1088 & 1.30 & 0.545 & 39.86 & 12.49 & 0.31 & 1.28 & $\mathrm{c}$ \\
\hline 40 & 183003.5 & -020045.1 & 7.58 & 0.65 & 28.0 & 0.0564 & 1.42 & 0.319 & 3.13 & 4.18 & 1.33 & 0.72 & c \\
\hline 41 & 183003.7 & -020612.3 & 7.62 & 0.75 & 24.8 & 0.0499 & 1.28 & 0.545 & 3.66 & 5.73 & 1.57 & 1.22 & $\mathrm{~s}$ \\
\hline 42 & 183003.8 & -01 5954.4 & 7.19 & 0.61 & 40.9 & 0.0823 & 1.47 & 0.468 & 9.98 & 8.12 & 0.81 & 0.74 & $\mathrm{n}$ \\
\hline 43 & 183004.0 & -020358.2 & 6.88 & 1.20 & 33.0 & 0.0664 & 2.01 & 0.579 & 13.74 & 8.16 & 0.59 & 1.94 & $\mathrm{c}$ \\
\hline 44 & 183004.3 & -020145.5 & 7.49 & 0.90 & 34.2 & 0.0687 & 1.38 & 0.503 & 9.76 & 7.27 & 0.74 & 1.24 & $\mathrm{c}$ \\
\hline 45 & 183005.0 & -020501.8 & 6.68 & 0.68 & 33.4 & 0.0672 & 1.30 & 0.384 & 3.94 & 5.62 & 1.43 & 0.54 & c \\
\hline 46 & 183005.3 & -020527.5 & 7.41 & 0.60 & 30.5 & 0.0613 & 1.08 & 0.455 & 5.30 & 5.88 & 1.11 & 0.95 & c \\
\hline 47 & 183005.4 & -020106.8 & 6.80 & 0.59 & 41.5 & 0.0834 & 1.18 & 0.697 & 5.59 & 12.88 & 2.30 & 0.40 & $\mathrm{c}$ \\
\hline 48 & 183006.1 & -020612.5 & 7.56 & 0.61 & 21.8 & 0.0439 & 1.02 & 0.541 & 2.44 & 5.00 & 2.05 & 1.20 & s \\
\hline 49 & 183006.2 & -020444.1 & 7.54 & 0.68 & 30.3 & 0.0610 & 1.24 & 0.460 & 5.29 & 5.91 & 1.12 & 0.96 & $\mathrm{c}$ \\
\hline 50 & 183006.8 & -020520.5 & 6.93 & 0.94 & 34.5 & 0.0695 & 1.33 & 0.477 & 9.09 & 6.98 & 0.77 & 1.12 & $\mathrm{c}$ \\
\hline 51 & 183007.0 & -02 0704.4 & 7.51 & 0.82 & 20.8 & 0.0419 & 1.29 & 0.391 & 1.37 & 3.55 & 2.59 & 0.77 & $\mathrm{~s}$ \\
\hline 52 & 183007.1 & -020315.8 & 7.58 & 0.70 & 34.9 & 0.0703 & 1.41 & 0.507 & 5.62 & 7.48 & 1.33 & 0.67 & $\mathrm{c}$ \\
\hline 53 & 183007.2 & -020105.6 & 7.76 & 0.61 & 36.5 & 0.0735 & 1.27 & 0.422 & 3.68 & 6.61 & 1.79 & 0.38 & $\mathrm{c}$ \\
\hline 54 & 183007.8 & -020626.2 & 7.14 & 0.59 & 24.7 & 0.0496 & 1.59 & 0.401 & 1.90 & 4.29 & 2.25 & 0.65 & $\mathrm{~s}$ \\
\hline 55 & 183007.9 & -02 0413.1 & 6.86 & 0.64 & 29.0 & 0.0584 & 1.09 & 0.244 & 2.45 & 3.81 & 1.55 & 0.51 & $\mathrm{c}$ \\
\hline 56 & 183008.1 & -020414.0 & 7.42 & 0.74 & 30.3 & 0.0610 & 1.42 & 0.392 & 4.99 & 5.18 & 1.04 & 0.91 & $\mathrm{c}$ \\
\hline 57 & 183008.1 & -020336.8 & 7.20 & 0.67 & 27.2 & 0.0548 & 1.64 & 0.320 & 2.83 & 4.06 & 1.44 & 0.71 & $\mathrm{c}$ \\
\hline 58 & 183008.2 & -020553.1 & 7.29 & 0.59 & 23.9 & 0.0481 & 1.84 & 0.460 & 2.67 & 4.67 & 1.75 & 0.99 & $\mathrm{~s}$ \\
\hline 59 & 183008.7 & -020514.3 & 7.30 & 0.64 & 22.8 & 0.0459 & 1.14 & 0.321 & 2.11 & 3.41 & 1.62 & 0.90 & c \\
\hline 60 & 183009.7 & -020544.3 & 6.73 & 0.92 & 26.7 & 0.0538 & 1.69 & 0.344 & 4.08 & 4.17 & 1.02 & 1.09 & $\mathrm{~s}$ \\
\hline
\end{tabular}


Table 4-Continued

\begin{tabular}{|c|c|c|c|c|c|c|c|c|c|c|c|c|c|}
\hline ID & $\begin{array}{c}\text { R.A. } \\
\text { (J2000.0) }\end{array}$ & $\begin{array}{c}\text { Decl. } \\
(\mathrm{J} 2000.0)\end{array}$ & $\begin{array}{c}V_{\mathrm{LSR}} \\
\left(\mathrm{km} \mathrm{s}^{-1}\right)\end{array}$ & $\begin{array}{c}T_{A, \text { peak }}^{*}{ }^{\mathrm{a}} \\
\quad(\mathrm{K})\end{array}$ & $\begin{array}{c}R_{\text {core }} \\
(\operatorname{arcsec})\end{array}$ & $\begin{array}{l}R_{\text {core }} \\
(\mathrm{pc})\end{array}$ & $\begin{array}{l}\text { Aspect } \\
\text { Ratio }\end{array}$ & $\begin{array}{c}\Delta v_{\text {core }} \\
\left(\mathrm{km} \mathrm{s}^{-1}\right)\end{array}$ & $\begin{array}{l}M_{\mathrm{LTE}} \\
\left(M_{\odot}\right)\end{array}$ & $\begin{array}{l}M_{\mathrm{vir}} \\
\left(M_{\odot}\right)\end{array}$ & $\alpha_{\mathrm{vir}}$ & $\begin{array}{c}\bar{n} \\
\left(10^{5} \mathrm{~cm}^{-3}\right)\end{array}$ & Area $^{b}$ \\
\hline 61 & 183009.9 & $\begin{array}{lll}-02 & 02 & 06.4\end{array}$ & 7.57 & 0.60 & 41.4 & 0.0834 & 1.30 & 0.403 & 3.96 & 7.22 & 1.82 & 0.28 & $\mathrm{c}$ \\
\hline 62 & 183010.8 & $\begin{array}{lll}-02 & 03 & 42.8\end{array}$ & 7.45 & 0.69 & 25.1 & 0.0505 & 1.58 & 0.324 & 3.34 & 3.77 & 1.13 & 1.08 & $\mathrm{c}$ \\
\hline 63 & 183011.0 & -020612.5 & 6.90 & 1.04 & 26.6 & 0.0535 & 1.56 & 0.544 & 7.02 & 6.13 & 0.87 & 1.90 & $\mathrm{~s}$ \\
\hline 64 & 183011.4 & -020425.6 & 7.07 & 0.61 & 29.2 & 0.0587 & 1.10 & 0.423 & 2.59 & 5.30 & 2.05 & 0.53 & $\mathrm{c}$ \\
\hline 65 & 183011.5 & -020653.4 & 6.91 & 0.90 & 27.4 & 0.0552 & 1.42 & 0.563 & 5.37 & 6.57 & 1.22 & 1.32 & s \\
\hline 66 & 183013.3 & -020417.7 & 7.50 & 0.66 & 26.0 & 0.0522 & 1.09 & 0.531 & 3.18 & 5.84 & 1.84 & 0.92 & $\mathrm{c}$ \\
\hline 67 & 183013.4 & $\begin{array}{lll}-02 & 07 & 00.7\end{array}$ & 6.17 & 0.59 & 30.2 & 0.0608 & 1.11 & 0.343 & 1.70 & 4.70 & 2.77 & 0.31 & $\mathrm{~s}$ \\
\hline 68 & 183013.5 & -020323.5 & 7.48 & 0.59 & 35.9 & 0.0722 & 1.32 & 0.476 & 4.19 & 7.23 & 1.73 & 0.46 & c \\
\hline 69 & 183013.7 & -020641.9 & 6.70 & 1.29 & 40.6 & 0.0817 & 1.08 & 0.436 & 14.77 & 7.56 & 0.51 & 1.12 & $\mathrm{~s}$ \\
\hline 70 & 183015.9 & -020443.6 & 7.49 & 0.66 & 30.1 & 0.0606 & 1.41 & 0.478 & 3.20 & 6.10 & 1.91 & 0.59 & c \\
\hline
\end{tabular}

Note. - Units of right ascension are hours, minutes, and seconds, and units of declination are degrees, arcminutes, and arcseconds. Taking into account the calibration error of the observed intensities of about $\pm 20 \%$, the derived LTE masses, virial ratios, and mean densities also have an error of at least about $\pm 20 \%$.

${ }^{\mathrm{a}} T_{A}^{*}$ denotes the peak antenna temperature of the isolated hyperfine component.

${ }^{\mathrm{b}}$ The symbols n, c, and s mean, respectively, the northern, central, and southern areas where the cores are located. 
Table 5. Summary of the Physical Properties of the $\mathrm{N}_{2} \mathrm{H}^{+}$cores

\begin{tabular}{|c|c|c|c|}
\hline Property & Minimum & Maximum & Mean $^{\mathrm{a}}$ \\
\hline \multicolumn{4}{|l|}{ whole area } \\
\hline$R_{\text {core }}(\mathrm{pc})$ & 0.0400 & 0.1088 & $0.0649 \pm 0.0154$ \\
\hline$d v_{\text {core }}\left(\mathrm{km} \mathrm{s}^{-1}\right)$ & 0.078 & 0.849 & $0.448 \pm 0.132$ \\
\hline$M_{\mathrm{LTE}}\left(\mathrm{M}_{\odot}\right)$ & 0.79 & 39.86 & $6.81 \pm 6.49$ \\
\hline$M_{\mathrm{vir}} / M_{\mathrm{LTE}}$ & 0.313 & 3.45 & $1.42 \pm 0.76$ \\
\hline $\bar{n}\left(\times 10^{5} \mathrm{~cm}^{-3}\right)$ & 0.25 & 3.15 & $0.96 \pm 0.58$ \\
\hline Aspect Ratio & 1.00 & 3.26 & $1.36 \pm 0.36$ \\
\hline \multicolumn{4}{|l|}{ north } \\
\hline$R_{\text {core }}(\mathrm{pc})$ & 0.0471 & 0.1052 & $0.0634 \pm 0.0158$ \\
\hline$d v_{\text {core }}\left(\mathrm{km} \mathrm{s}^{-1}\right)$ & 0.275 & 0.644 & $0.423 \pm 0.100$ \\
\hline$M_{\mathrm{LTE}}\left(\mathrm{M}_{\odot}\right)$ & 4.59 & 23.62 & $9.83 \pm 5.57$ \\
\hline$M_{\mathrm{vir}} / M_{\mathrm{LTE}}$ & 0.344 & 0.86 & $0.68 \pm 0.18$ \\
\hline $\bar{n}\left(\times 10^{5} \mathrm{~cm}^{-3}\right)$ & 0.74 & 3.15 & $1.64 \pm 0.67$ \\
\hline Aspect Ratio & 1.01 & 1.77 & $1.33 \pm 0.21$ \\
\hline \multicolumn{4}{|l|}{ center } \\
\hline$R_{\text {core }}(\mathrm{pc})$ & 0.0400 & 0.1088 & $0.0693 \pm 0.0150$ \\
\hline$d v_{\text {core }}\left(\mathrm{km} \mathrm{s}^{-1}\right)$ & 0.244 & 0.849 & $0.464 \pm 0.141$ \\
\hline$M_{\mathrm{LTE}}\left(\mathrm{M}_{\odot}\right)$ & 2.01 & 39.86 & $7.16 \pm 7.12$ \\
\hline$M_{\mathrm{vir}} / M_{\mathrm{LTE}}$ & 0.313 & 3.17 & $1.43 \pm 0.67$ \\
\hline $\bar{n}\left(\times 10^{5} \mathrm{~cm}^{-3}\right)$ & 0.25 & 2.49 & $0.81 \pm 0.45$ \\
\hline Aspect Ratio & 1.00 & 2.45 & $1.30 \pm 0.27$ \\
\hline \multicolumn{4}{|l|}{ south } \\
\hline$R_{\text {core }}(\mathrm{pc})$ & 0.0419 & 0.0817 & $0.0546 \pm 0.0101$ \\
\hline$d v_{\text {core }}\left(\mathrm{km} \mathrm{s}^{-1}\right)$ & 0.078 & 0.563 & $0.426 \pm 0.122$ \\
\hline$M_{\mathrm{LTE}}\left(\mathrm{M}_{\odot}\right)$ & 0.79 & 14.77 & $3.61 \pm 3.27$ \\
\hline$M_{\mathrm{vir}} / M_{\mathrm{LTE}}$ & 0.512 & 3.45 & $1.97 \pm 0.79$ \\
\hline $\bar{n}\left(\times 10^{5} \mathrm{~cm}^{-3}\right)$ & 0.31 & 1.90 & $0.85 \pm 0.43$ \\
\hline Aspect Ratio & 1.01 & 3.26 & $1.54 \pm 0.54$ \\
\hline
\end{tabular}

${ }^{a}$ With standard deviation 


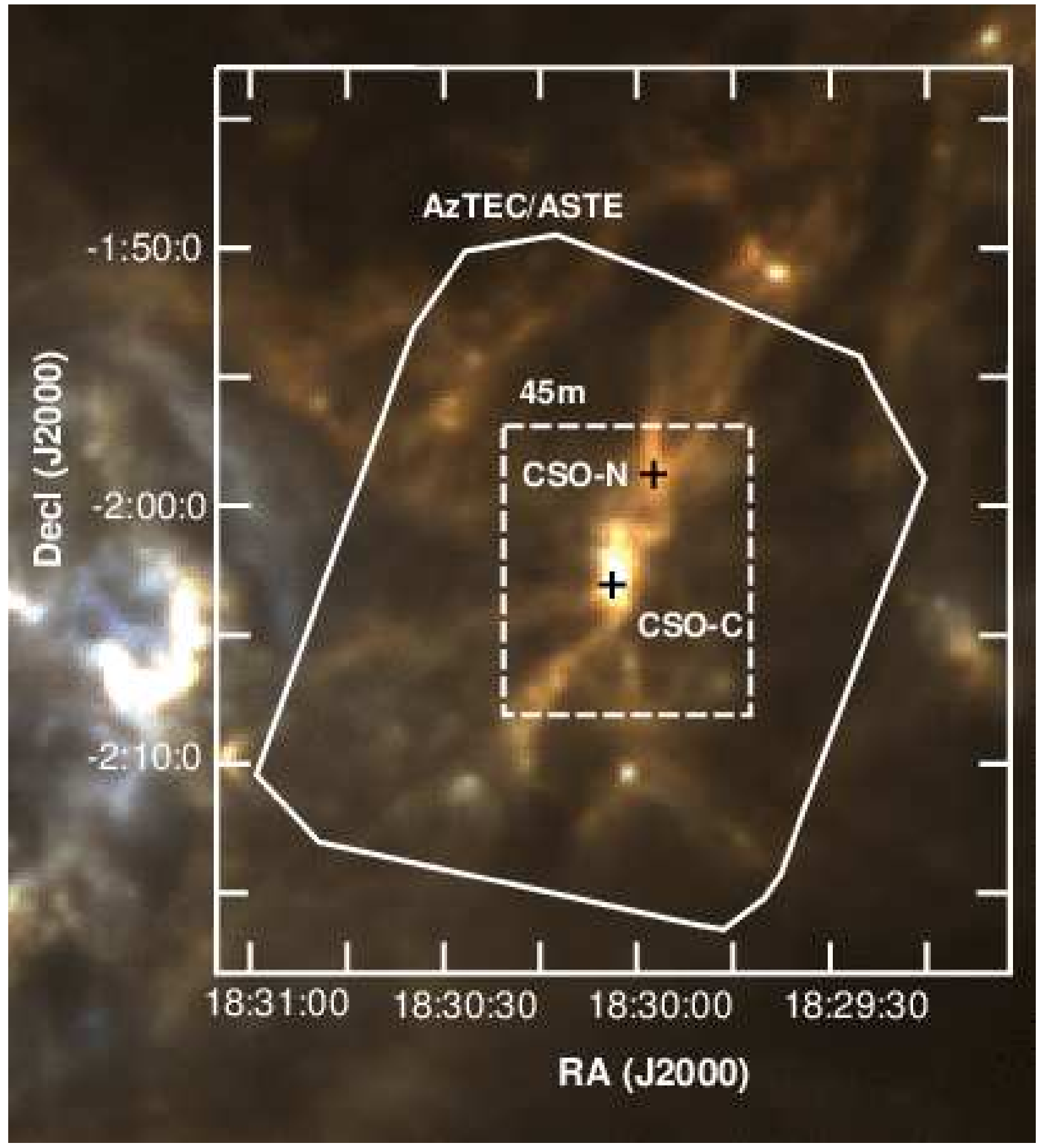

Fig. 1.- Three-color Herschel image of Serpens South with SPIRE $250 \mu \mathrm{m}$ (blue), $350 \mu \mathrm{m}$ (green), and $500 \mu \mathrm{m}(\mathrm{red})$. The observed areas are the dashed box and the area enclosed by solid lines for the NRO 45-m N $\mathrm{N}_{2} \mathrm{H}^{+}$observations and AzTEC/ASTE $1.1 \mathrm{~mm}$ observations, respectively. Two points of the CSO observations are indicated with the crosses. The eastern bright area outside the panel is the dense gas around the W40 HII region. 

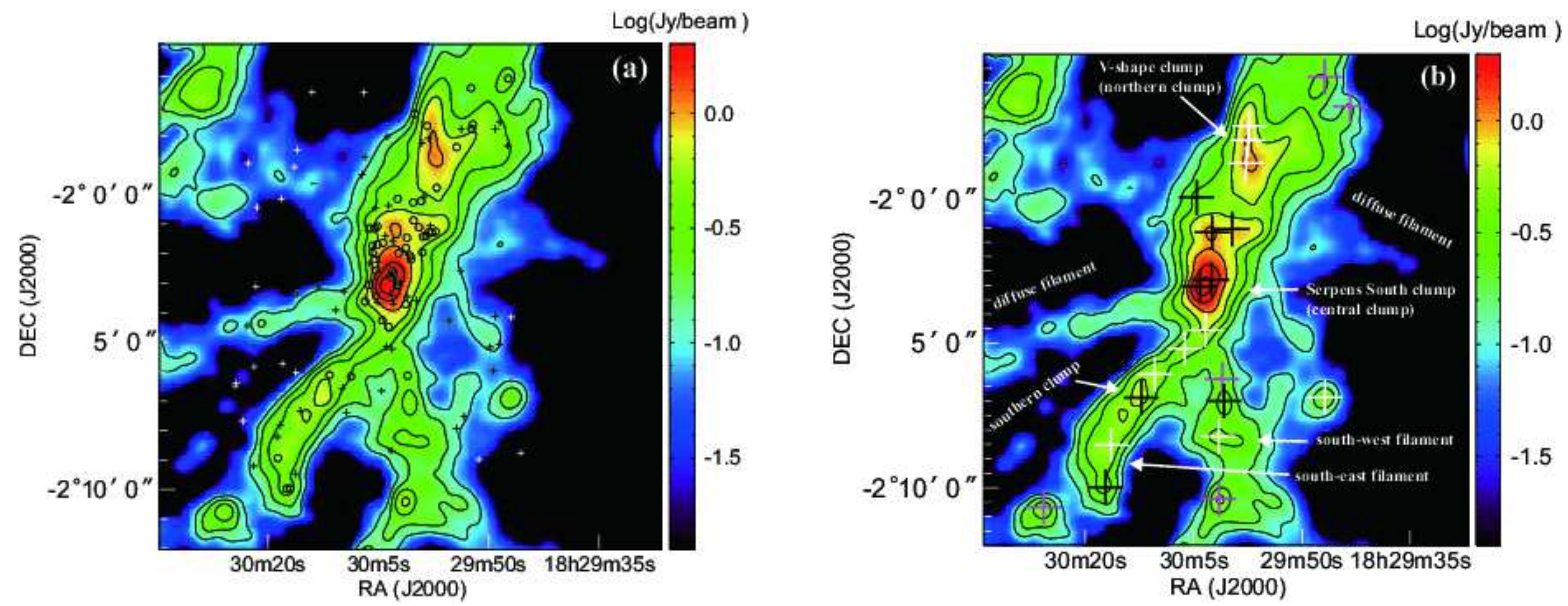

Fig. 2.- (a) $1.1 \mathrm{~mm}$ continuum emission map taken by the AzTEC camera on the ASTE telescope. The circles and crosses indicate the positions of the Spitzer Class I and II YSOs, respectively. (b) Same as panel (a) but with the positions of the starless cores, Class 0, and Class I sources newly identified by the Herschel observations (Bontemps et al. 2010). The white, black, and magenta crosses are the starless cores, Class 0, and Class I sources, respectively. The names of several features discussed in the main text are designated in the panel. For both the panels, the contours start at $-1.0(=10 \sigma)$ with intervals of 0.25 on a logarithmic scale. 

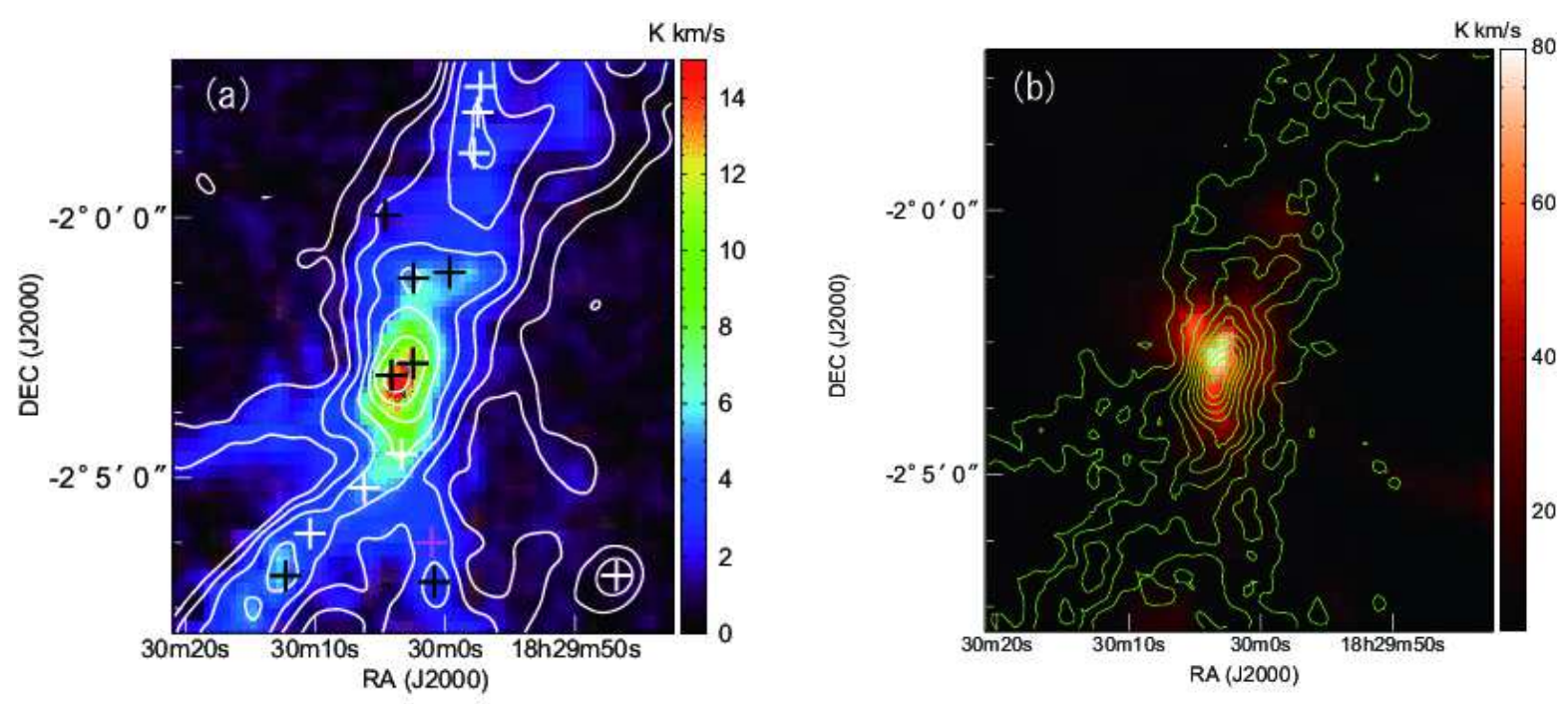

Fig. 3.- (a) $\mathrm{N}_{2} \mathrm{H}^{+}(J=1-0)$ intensity map integrated over the seven components of the hyperfine multiplet, taken with the Nobeyama 45-m telescope toward Serpens South. The contours of the AzTEC $1.1 \mathrm{~mm}$ dust continuum emission are overlaid on the image. The contours start from -1.0 with an interval of 0.25 in a logarithmic scale. The white, black, and magenta crosses indicate the positions of the starless cores, Class 0, and Class I sources newly identified by the Herschel observations (Bontemps et al. 2010). (b) $\mathrm{N}_{2} \mathrm{H}^{+}(J=1-0)$ intensity contour map overlaid on the ${ }^{12} \mathrm{CO}(J=3-2)$ integrated intensity map in the range of $v_{\mathrm{LSR}}=-2 \mathrm{~km} \mathrm{~s}^{-1}$ to $+15 \mathrm{~km} \mathrm{~s}^{-1}$. The contour level starts from $2 \mathrm{~K} \mathrm{~km} \mathrm{~s}^{-1}$ with an interval of $1.5 \mathrm{~K} \mathrm{~km} \mathrm{~s}^{-1}$. 


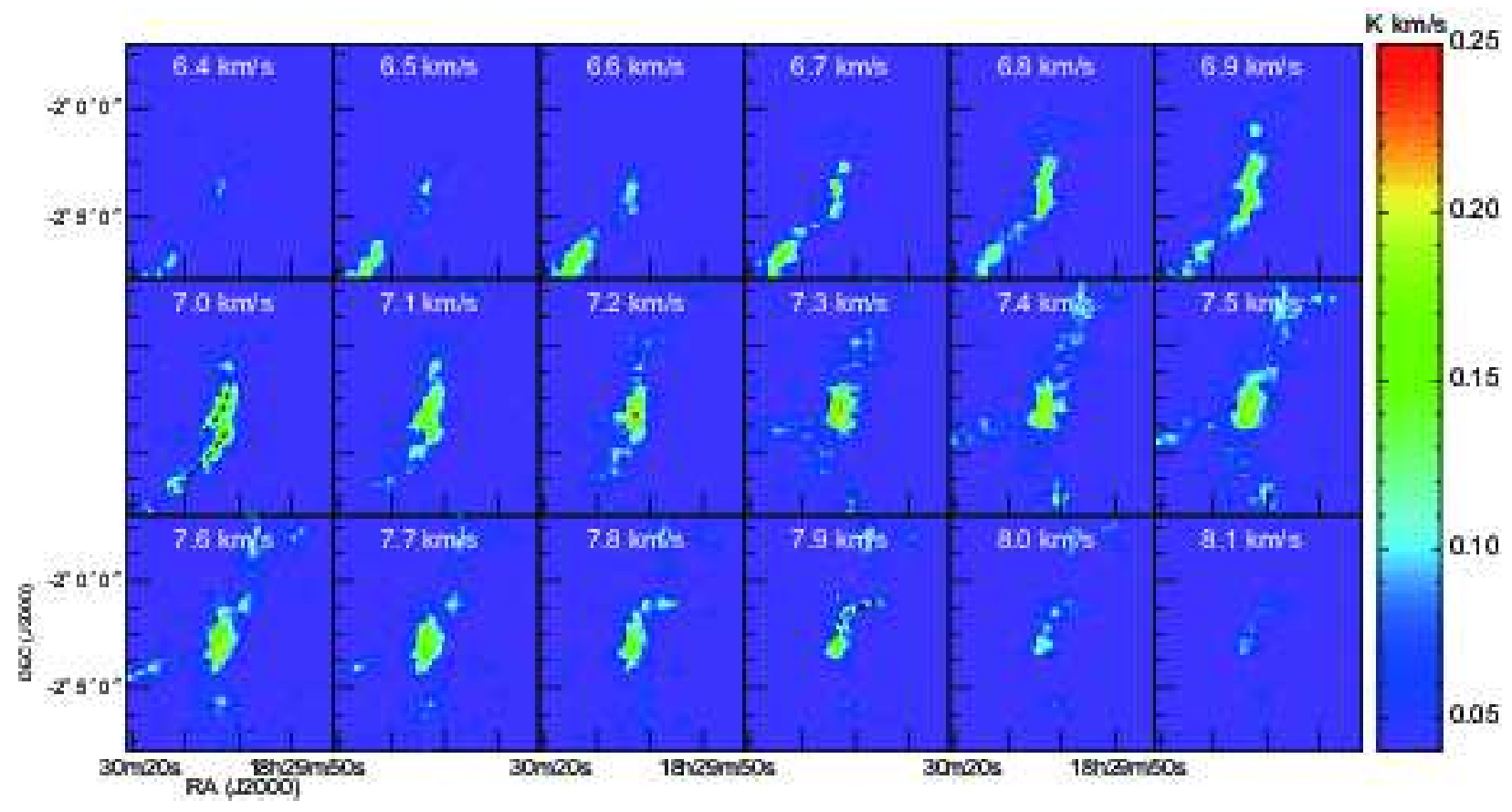

Fig. 4. $-\mathrm{N}_{2} \mathrm{H}^{+}(J=1-0)$ velocity channel maps of the isolated component of the hyperfine multiplet, taken with the Nobeyama 45-m telescope toward Serpens South. The reference frequency is set to the rest frequency of the isolated component to make the maps. Some arc-like or filamentary structures are highlighted by the dashed curves. 


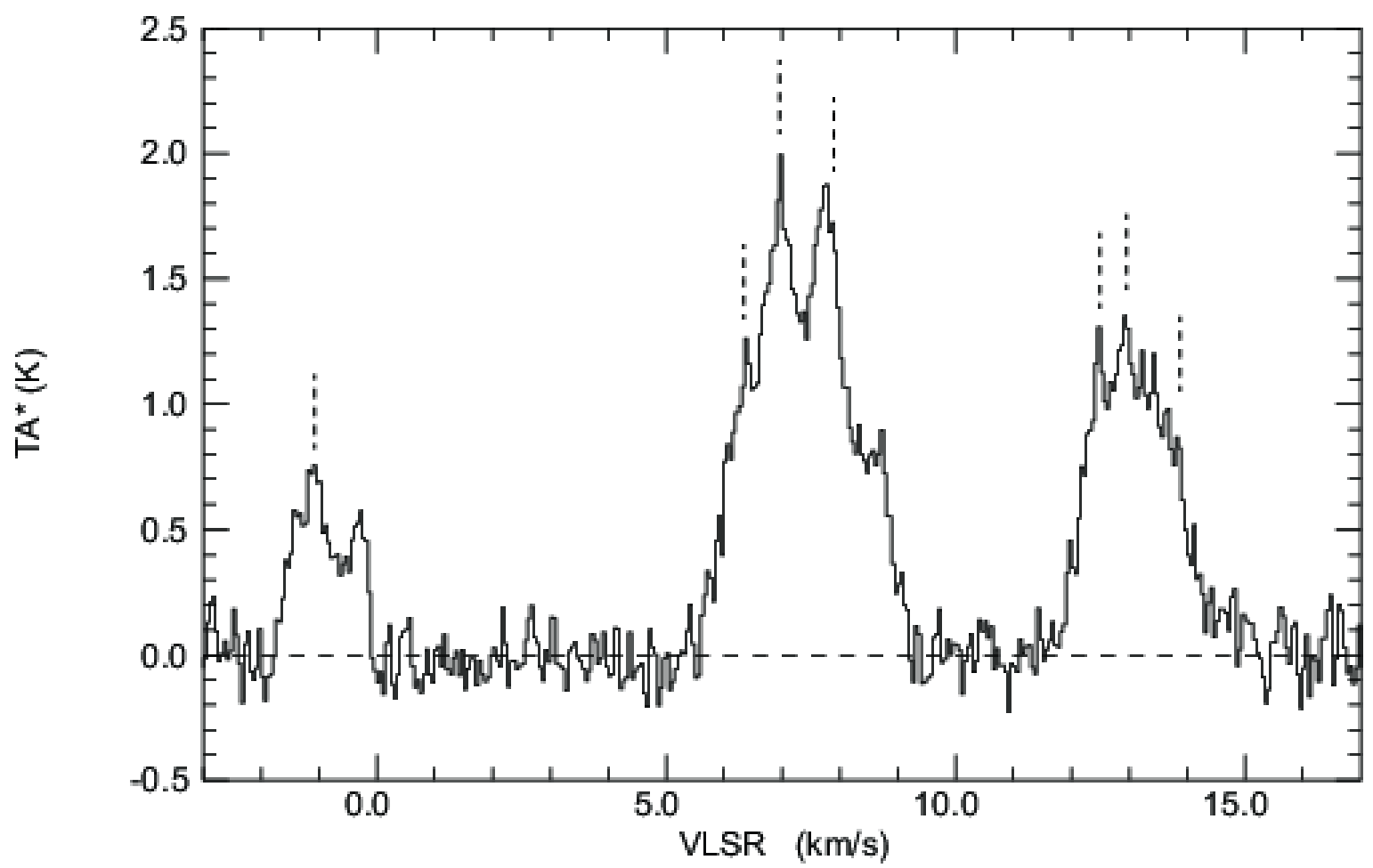

Fig. 5. $-\mathrm{N}_{2} \mathrm{H}^{+}(J=1-0)$ line profile averaged in the $30^{\prime \prime} \times 30^{\prime \prime}$ area centered on the position (R.A. [J2000], Decl. [J2000] $)=(18: 30: 4.4,-02: 04: 23.5)$. The velocity resolution is set to $0.05 \mathrm{~km} \mathrm{~s}^{-1}$. The reference frequency is set to the rest frequency of the main component of $F_{1} F \rightarrow F_{1}^{\prime} F^{\prime}=23 \rightarrow 12$, 93.173777 GHz, to make the plot. 

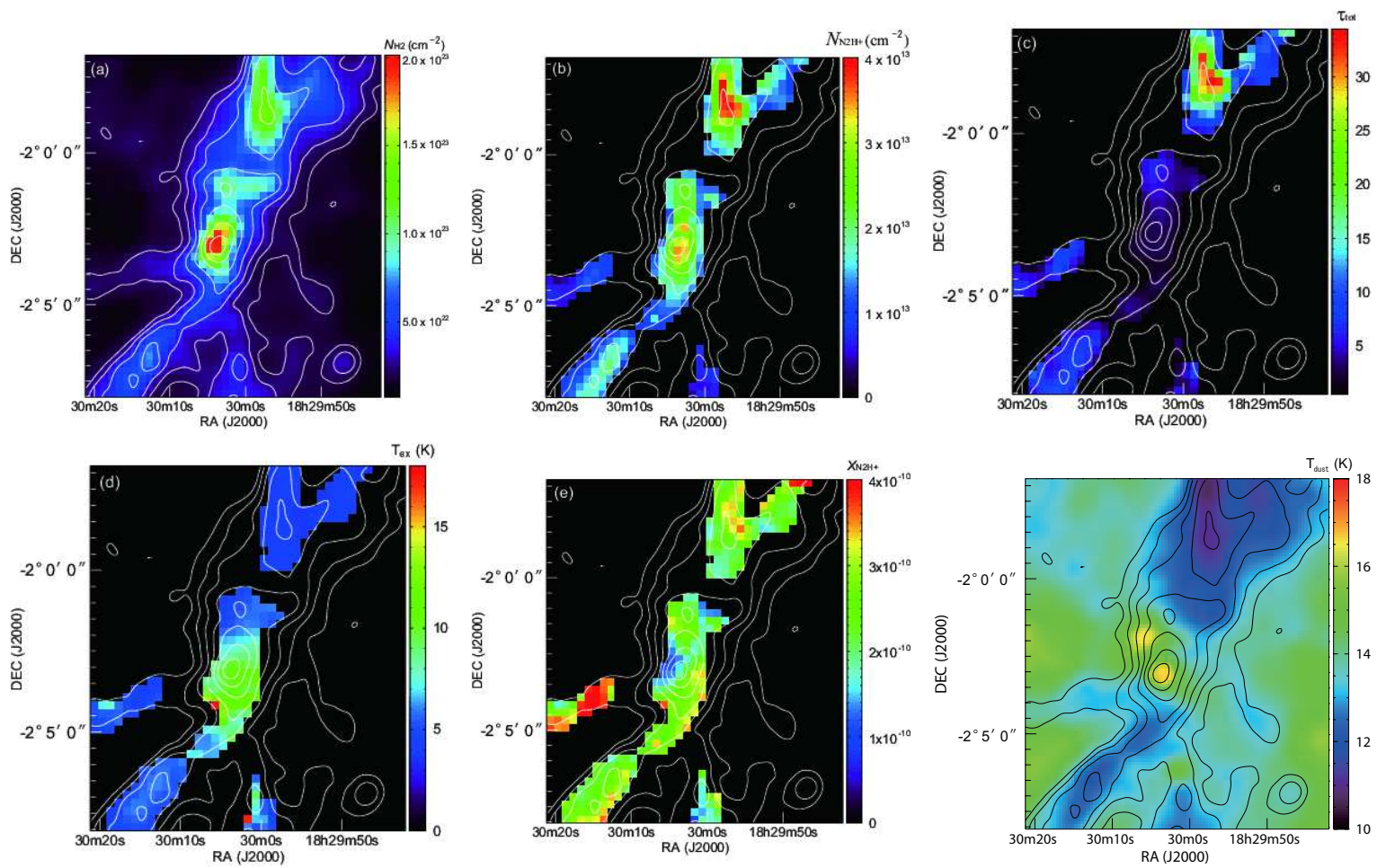

Fig. 6.- (a) $\mathrm{H}_{2}$ column density distribution $\left(N_{\mathrm{H}_{2}}\right)$ obtained from the SED fitting of the Herschel data. (b) $\mathrm{N}_{2} \mathrm{H}^{+}$column density distribution $\left(N_{\mathrm{N}_{2} \mathrm{H}^{+}}\right)$obtained from the hyperfine fitting of the $\mathrm{N}_{2} \mathrm{H}^{+}$data cube. (c) total optical depth distribution obtained from the hyperfine fitting of the $\mathrm{N}_{2} \mathrm{H}^{+}$data cube. (d) excitation temperature distribution obtained from the hyperfine fitting of the $\mathrm{N}_{2} \mathrm{H}^{+}$data cube toward Serpens South. (e) $\mathrm{N}_{2} \mathrm{H}^{+}$fractional abundance distribution $\left(X_{\mathrm{N}_{2} \mathrm{H}^{+}}=\right.$ $N_{\mathrm{N}_{2} \mathrm{H}^{+}} / N_{\mathrm{H}_{2}}$ ) toward Serpens South. (f) Dust temperature distribution ( $\left.T_{\text {dust }}\right)$ obtained from the SED fitting of the Herschel data. For all the panels, the contour lines indicate the the $1.1 \mathrm{~mm}$ dust continuum emission distribution. The contours start from -1.0 with an interval of 0.25 in a logarithmic scale. 


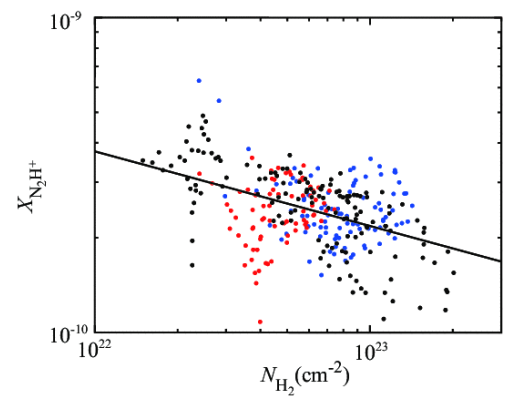

Fig. 7.- Fractional abundance of $\mathrm{N}_{2} \mathrm{H}^{+}$relative to $\mathrm{H}_{2}$ against the $\mathrm{H}_{2}$ column density in Serpens South. The $\mathrm{H}_{2}$ column densities are derived by the SED fitting of the Herschel data. The solid line shows the best-fit power-law of $X_{\mathrm{N}_{2} \mathrm{H}^{+}}=2.2 \times 10^{-10}\left(N_{\mathrm{H}_{2}} / 10^{23} \mathrm{~cm}^{-2}\right)^{-0.237}$. The blue, red, and black dots indicate the values at the pixels located in the northern, central, and southern areas, respectively. The northern, central, and southern areas are defined as follows: north (Decl. [J2000] $\geq-02: 02: 02.4)$, center (-02:02:02.4 $\geq$ Decl. [J2000] $\geq-02: 05: 32.5)$, and south (Decl. [J2000] $\leq$ $-02: 05: 32.5)$. 

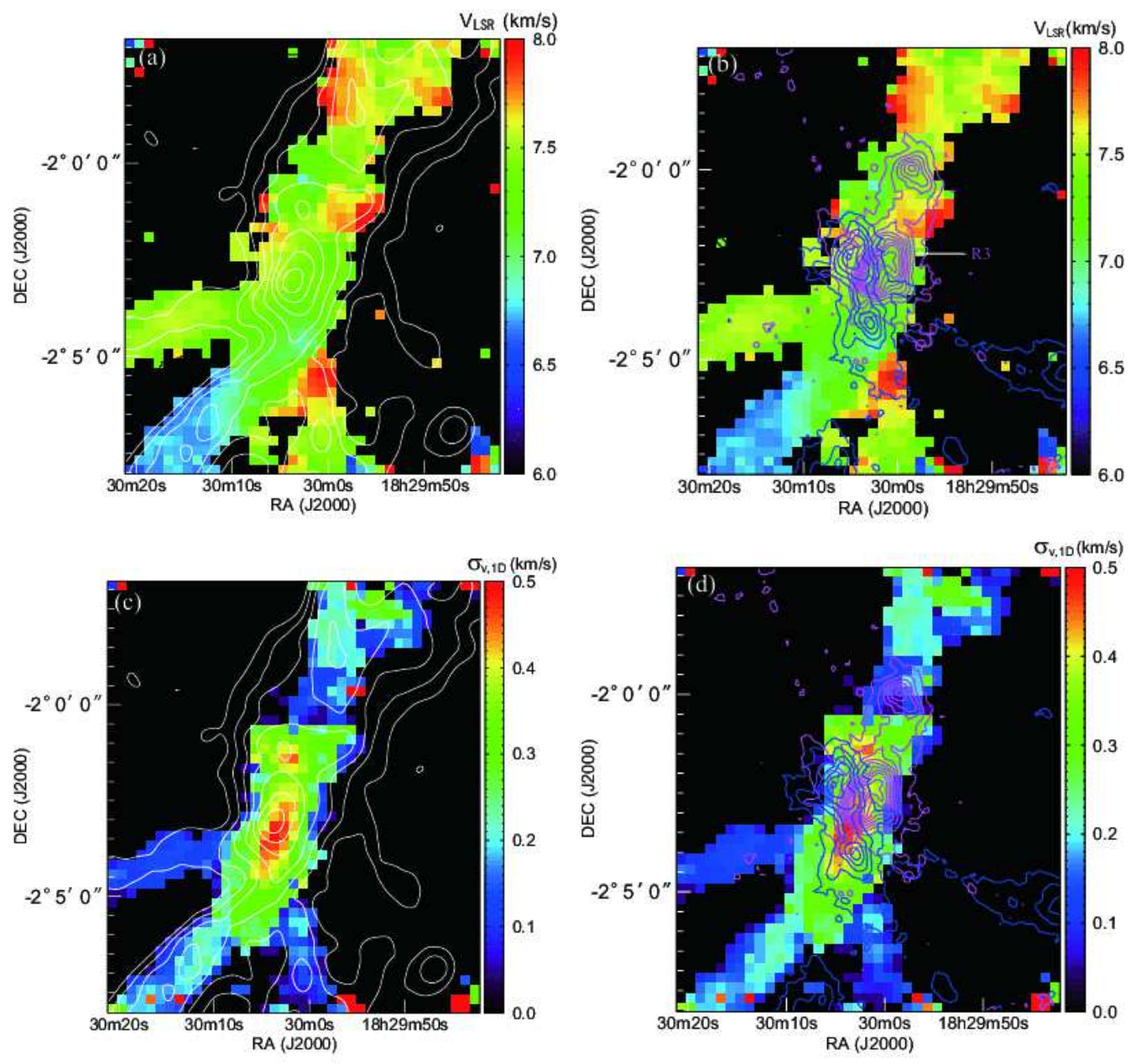

Fig. 8.- (a) the centroid velocity distribution toward Serpens South. (b) same as panel (a) but with the $\mathrm{CO}(J=3-2)$ outflow lobes overlaid. (c) the distribution of the one-dimensional velocity dispersion toward Serpens South. (d) same as panel (c) but with the CO $(J=3-2)$ outflow lobes overlaid. The centroid velocity and $1 \mathrm{D}$ velocity dispersion are calculated in the range from 6 $\mathrm{km} \mathrm{s}^{-1}$ to $9.5 \mathrm{~km} \mathrm{~s}^{-1}$ by integrating all the channels with the intensity larger than $0.3 \mathrm{~K}(\simeq 4 \sigma)$. In panels (b) and (d), the blue contours represent blueshifted ${ }^{12} \mathrm{CO}$ gas and magenta contours represent redshifted ${ }^{12} \mathrm{CO}$ gas. The blue and magenta contour levels go up in $6 \mathrm{~K} \mathrm{~km} \mathrm{~s}^{-1}$ steps, starting from $3 \mathrm{~K} \mathrm{~km} \mathrm{~s}^{-1}$. The integration ranges are -9.75 to $3.75 \mathrm{~km} \mathrm{~s}^{-1}$ for blueshifted gas and 11.25 to $29.25 \mathrm{~km} \mathrm{~s}^{-1}$ for redshifted gas. See Nakamura et al. (2011) in more detail. We note that the isothermal sound speed is about $0.23 \mathrm{~km} \mathrm{~s}^{-1}$ at the temperature of $15 \mathrm{~K}$. Therefore, the pixels having green, yellow, or red color are roughly supersonic, the pixels having cyan are transonic, and the pixels having blue are subsonic. 


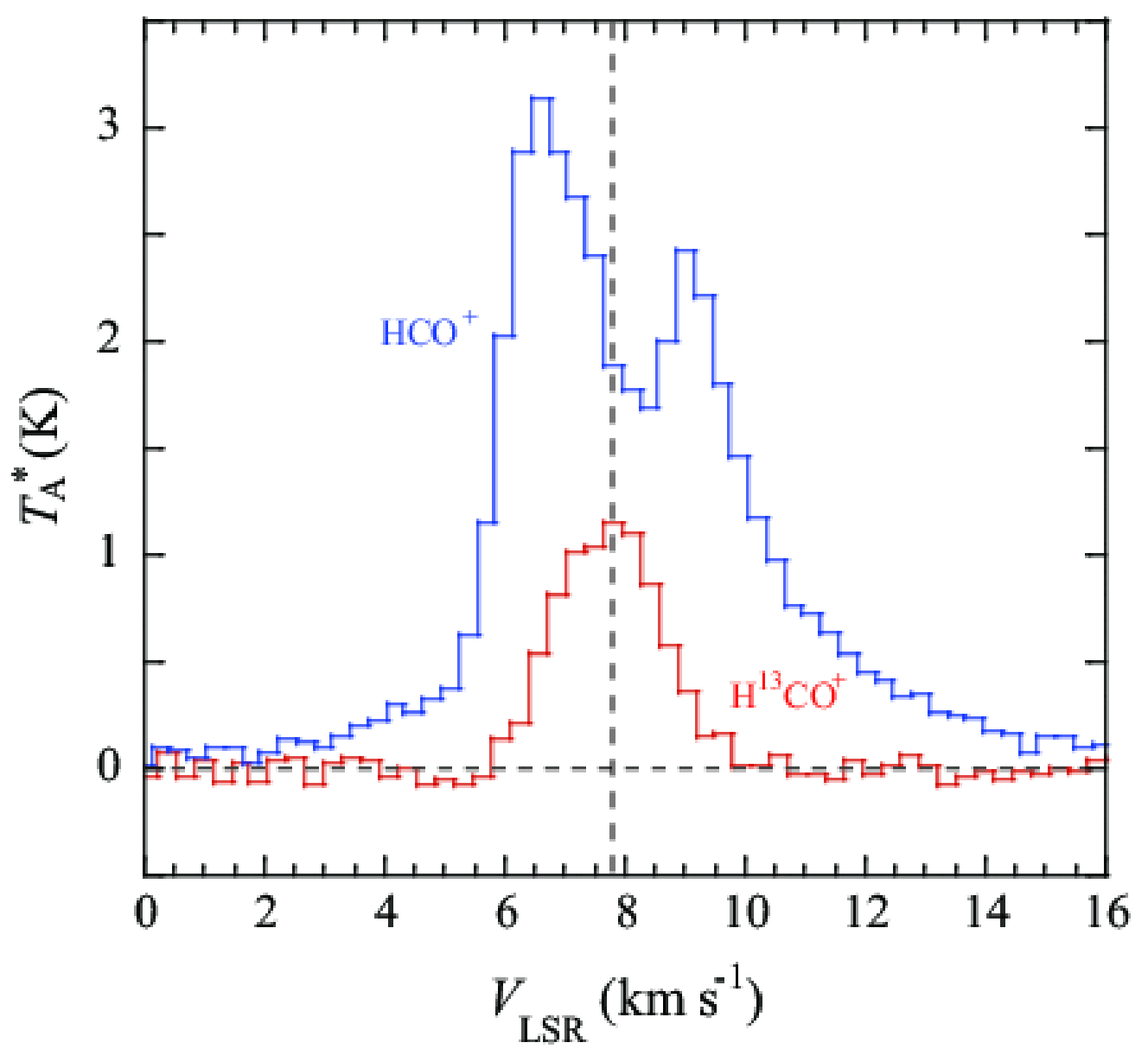

Fig. 9. $-\mathrm{HCO}^{+}(J=3-2$, blue $)$ and $\mathrm{H}^{13} \mathrm{CO}^{+}(J=3-2$, red $)$ line profiles toward the Serpens South clump at the position of (R.A. [J2000], Dec. [J2000]) $=(18: 30: 3.80,-2: 03: 03.9)$. The FWHM beam size of the CSO 10.4-m telescope was $30^{\prime \prime}$. The optically-thick $\mathrm{HCO}^{+}(J=3-2)$ line shows a clear blue-skewed profile, i.e., infall signature, with prominent blueshifted and redshifted wings that are probably caused by the powerful molecular outflows. On the other hand, the optically-thin $\mathrm{H}^{13} \mathrm{CO}^{+}(J=3-2)$ line shows a single-peak profile. The dashed line indicates the value of $V_{\mathrm{LSR}}$ at the $\mathrm{H}^{13} \mathrm{CO}^{+}(J=3-2)$ emission peak. 


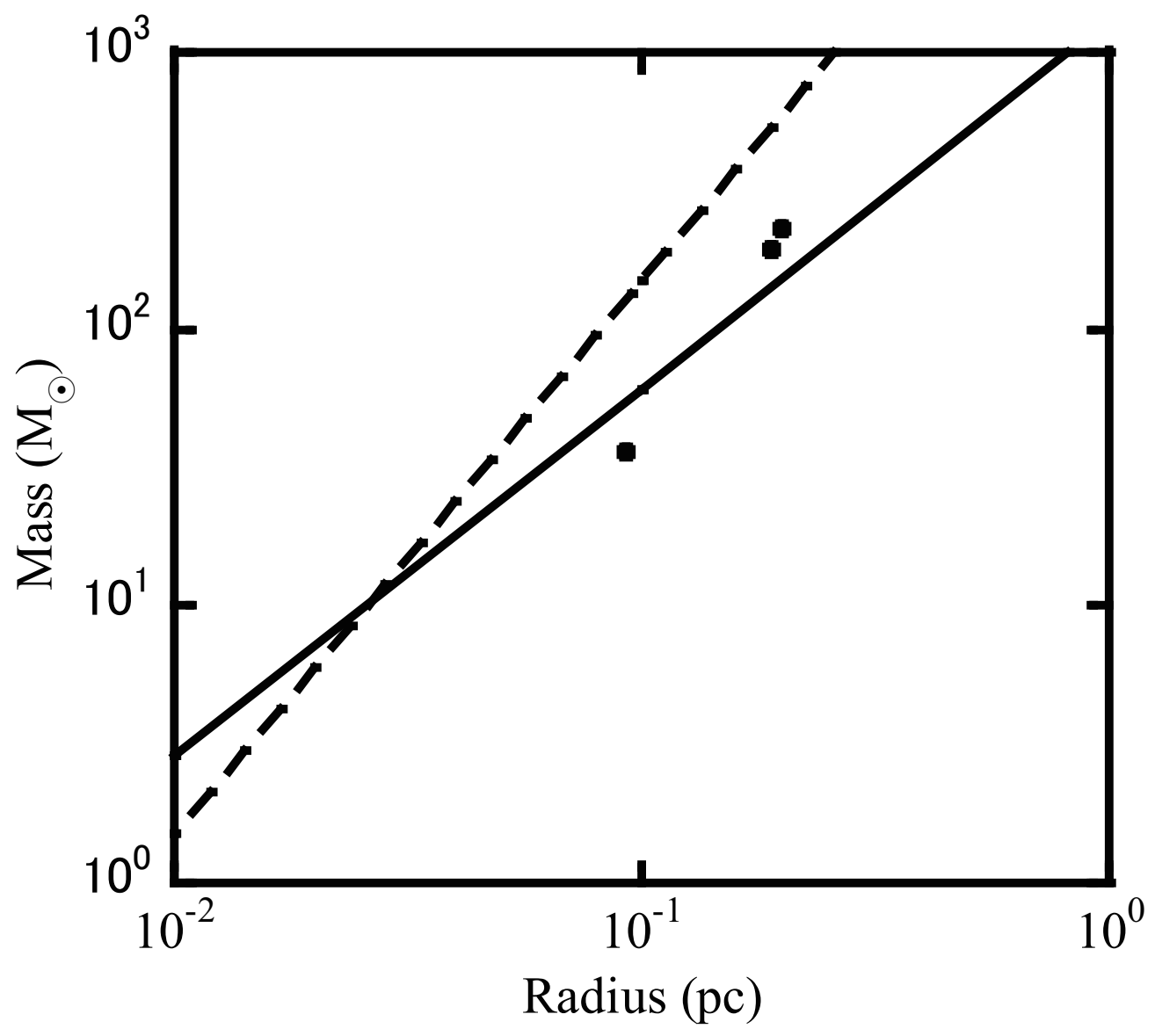

Fig. 10.- Clump mass-radius relation for the three Serpens South clumps. The solid and dashed lines indicate the criteria for the massive star formation by Kauffmann \& Pillai (2010) and Krumholz \& McKee (2008), respectively. 
(a) Fragmentation phase

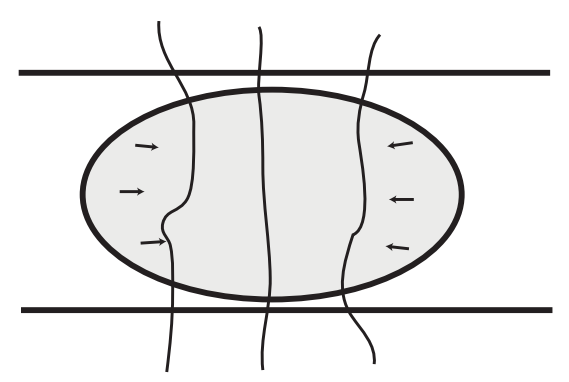

(c) Early protocluster phase

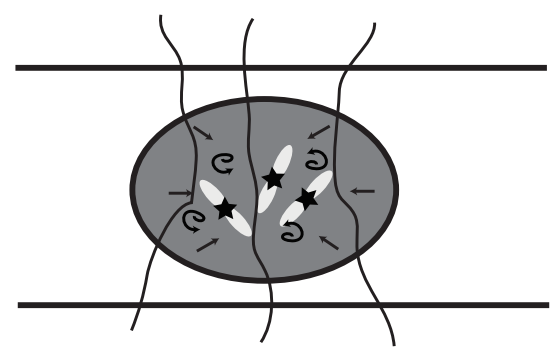

(b) Pre-protocluster phase

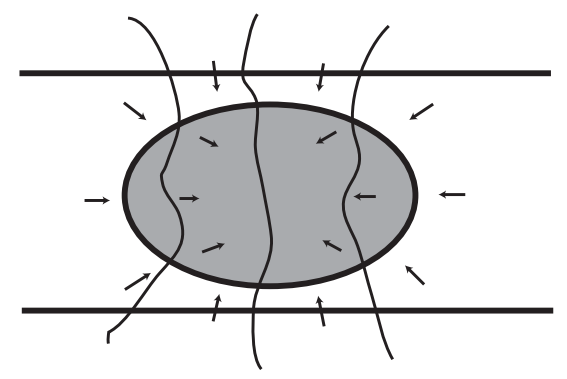

(d) Quasi-virial equilibrium phase

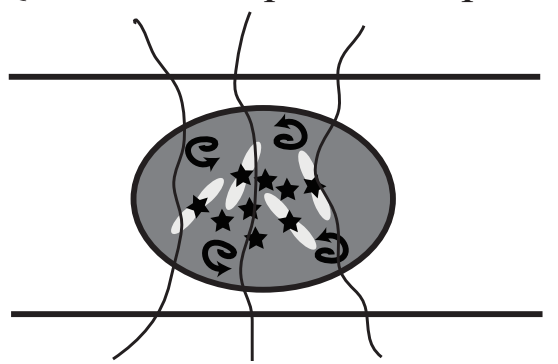

Fig. 11. - Magnetically-regulated cluster formation in Serpens South. (a) When the initial filament is supported by the strong magnetic field, the fragmentation is driven by the ambipolar diffusion to form dense clumps. (b) a dense clump grows in mass due to the mass infall along and/or across the magnetic field lines. Or, the merging of the clumps along the filament axis may play a role in increasing the clump mass. Although the accretion or merging supplies turbulent motions in the clump, the internal motions are relatively small. The virial ratio of such a clump may be only a few tenth unless the magnetic support is not taken into account. This stage is refered to as "preprotocluster" stage. (c) active cluster formation is initiated in the clump. (d) protostellar outflows inject additional momentum in the ambient material and the clump as a whole is supported by the outflow-driven turbulence. 

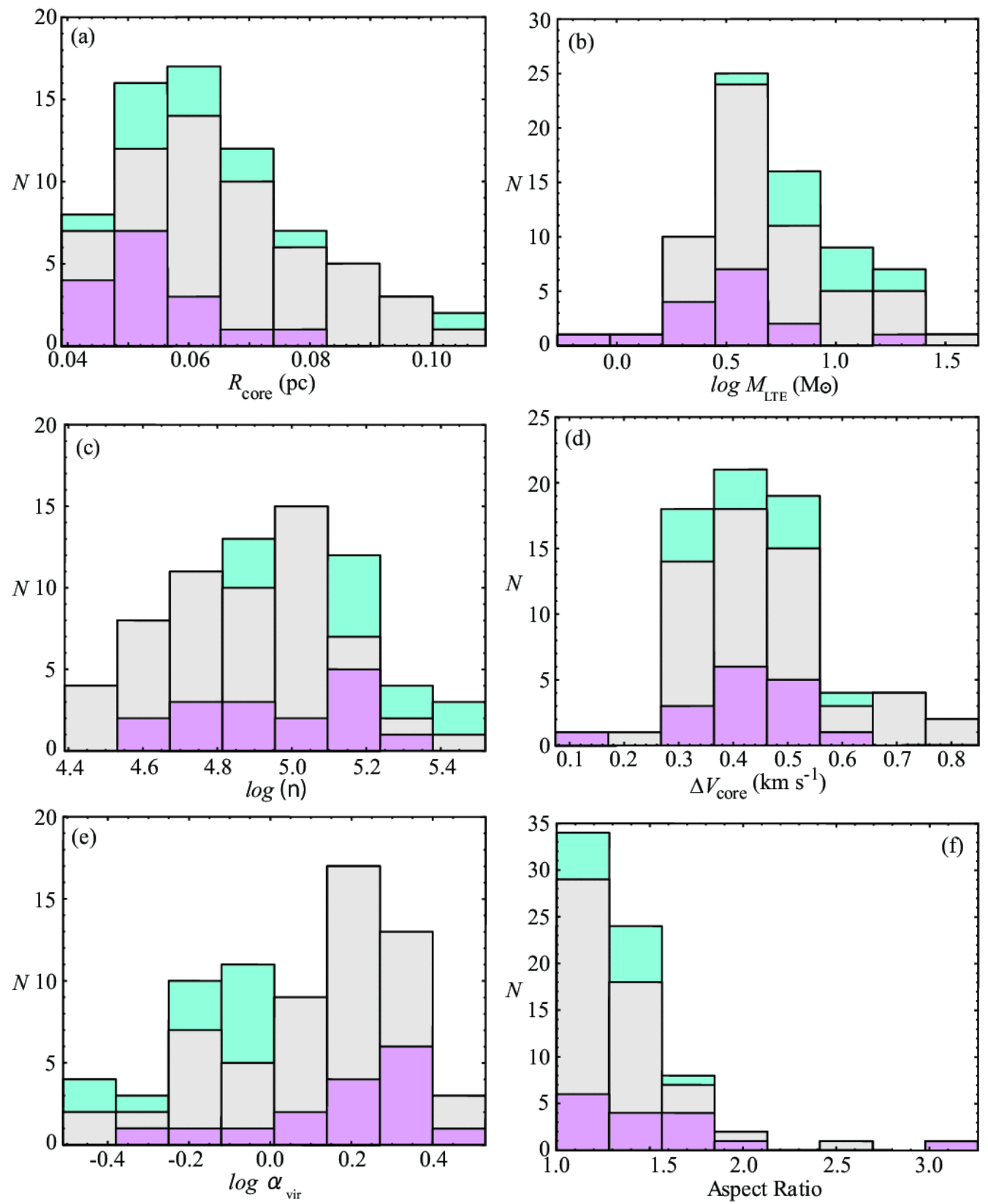

Fig. 12. - Histograms of (a) radius $\left(R_{\text {core }}\right)$, (b) LTE mass $\left(M_{\text {LTE }}\right)$, (c) mean density $(\bar{n})$, (d) FWHM line width $\left(\Delta V_{\text {core }}\right)$, (e) virial ratio $\left(\alpha_{\text {vir }}\right)$, and (f) aspect ratio of the $\mathrm{N}_{2} \mathrm{H}^{+}$cores. The cyan, grey, and magenta histograms indicate the cores located in the northern, central, and southern areas, respectively. 

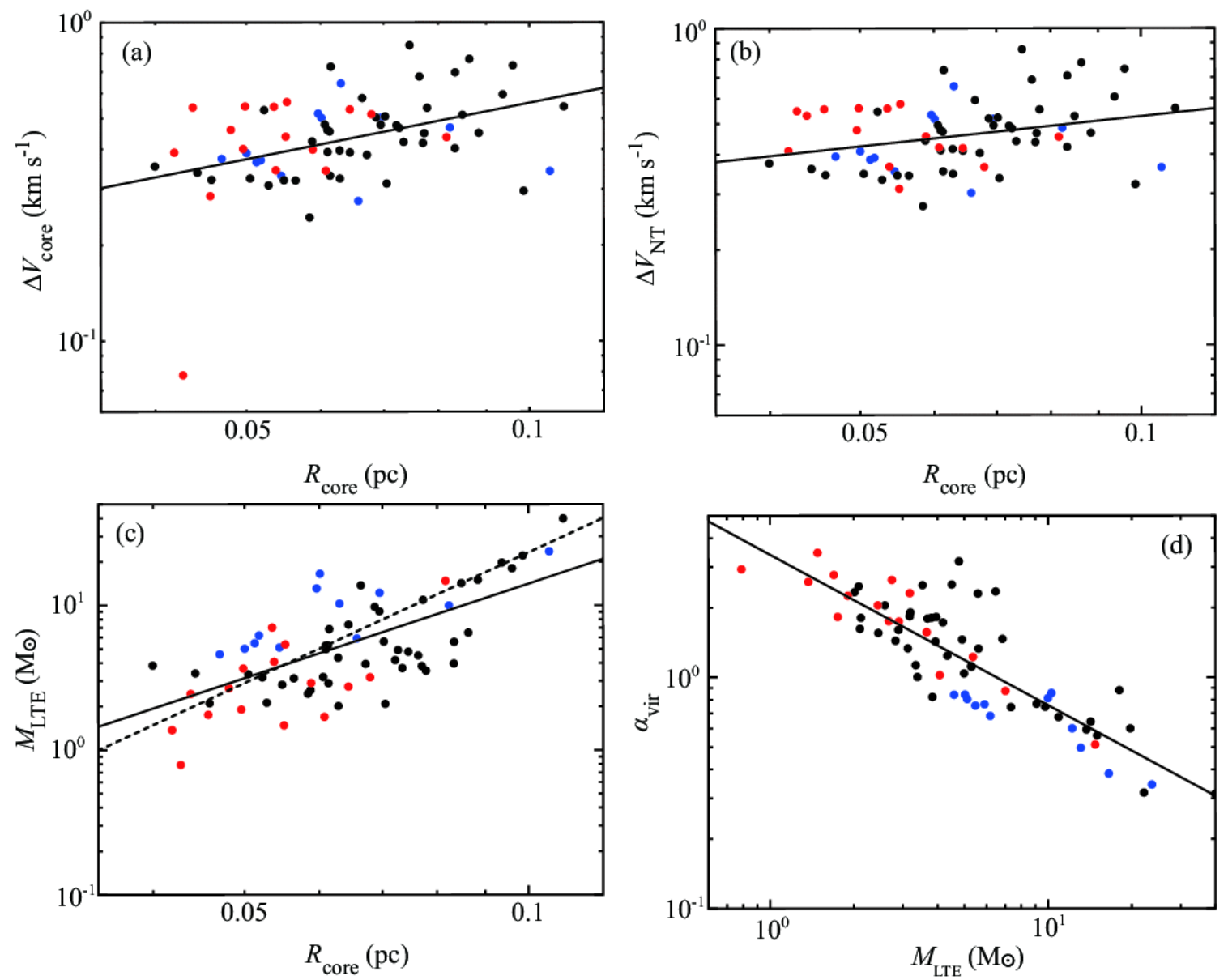

Fig. 13. - (a) Line-width-radius relation, (b) nonthermal line-width-radius relatoin, (c) mass-radius relation, and (d) virial-ratio-mass relation of the $\mathrm{N}_{2} \mathrm{H}^{+}$cores. The blue, red, and black circles are the same as those of Figure 7. 